\title{
1 Cryo-EM structures reveal high-resolution mechanism of a DNA polymerase sliding clamp loader
}

3

4 Christl Gaubitz ${ }^{a}$, Xingchen Liu ${ }^{a}$, Joshua Pajak ${ }^{a}$, Nicholas P. Stone ${ }^{a}$, Janelle A. Hayes ${ }^{a}$, Gabriel Demo $^{\text {b }}$, Brian A Kelch ${ }^{\text {an }}$

$7{ }^{\text {a }}$ Department of Biochemistry and Molecular Pharmacology, University of Massachusetts Chan Medical School, 8 Worcester MA

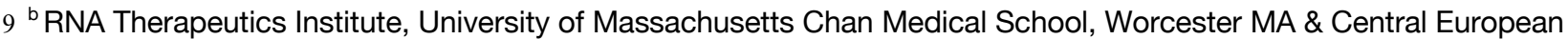
10 Institute of Technology, Masaryk University, Brno, Czech Republic

11 I Correspondence: brian.kelch@umassmed.edu, address: 364 Plantation St., Worcester MA 01605, phone: 508$12856-8322$

\section{SUMMARY}

14 Sliding clamps are ring-shaped protein complexes that are integral to the DNA replication machinery of all life. 15 Sliding clamps are opened and installed onto DNA by clamp loader AAA+ ATPase complexes. However, how 16 a clamp loader opens and closes the sliding clamp around DNA is still unknown. Here, we describe structures 17 of the S. cerevisiae clamp loader Replication Factor C (RFC) bound to its cognate sliding clamp Proliferating 18 Cell Nuclear Antigen (PCNA) en route to successful loading. RFC first binds to PCNA in a dynamic, closed 19 conformation that blocks both ATPase activity and DNA binding. RFC then opens the PCNA ring through a 20 large-scale 'crab-claw' expansion of both RFC and PCNA that explains how RFC prefers initial binding of PCNA 21 over DNA. Next, the open RFC:PCNA complex binds DNA and interrogates the primer-template junction using 22 a surprising base-flipping mechanism. Our structures indicate that initial PCNA opening and subsequent 23 closure around DNA do not require ATP hydrolysis, but are driven by binding energy. ATP hydrolysis, which is 24 necessary for RFC release, is triggered by interactions with both PCNA and DNA, explaining RFC's switch-like 25 ATPase activity. Our work reveals how a AAA+ machine undergoes dramatic conformational changes for 26 achieving binding preference and substrate remodeling. 27

28 Keywords: sliding clamp, DNA replication, AAA+, ATPase, clamp loader

\section{INTRODUCTION}

30 In all known cellular life, DNA replication is coordinated by ring31 shaped sliding clamp proteins that wrap around DNA to activate 32 DNA polymerases and other factors (Moldovan et al., 2007). 33 Sliding clamps are regulated by their presence on DNA, which in 34 turn is governed by clamp loaders that open the sliding clamp ring 35 and place it onto DNA (Kelch, 2016). The clamp loader of 36 eukaryotes Replication Factor $\underline{\mathrm{C}}$ (RFC) installs the sliding clamp 37 Proliferating Cell Nuclear Antigen (PCNA) in a coordinated and 38 stepwise fashion (Kelch, 2016). First, RFC binds ATP, which is a 39 prerequisite for tight binding to PCNA (Sakato et al., 2012). Next, 40 RFC binds to PCNA, and then opens the PCNA ring. This open 41 ternary complex is now competent to bind to primer-template $(p / t)$ 42 DNA (double-stranded DNA with a single-stranded 5' overhang). 43 Primer-template binding to the ternary complex triggers ATP 44 hydrolysis in the clamp loader, followed by sliding clamp closure 45 and ultimately release of the clamp loader complex (Chen et al., 46 2009). Therefore, RFC has two macromolecular substrates, PCNA 47 and $p / t-D N A$, that must bind sequentially. Yet how clamp loaders 48 achieve this strict sequential order remains unknown.

49 Clamp loaders are members of the ATPases associated with 50 various cellular activities (AAA+) family (Erzberger and Berger, 51 2006). Most members of this family function as ringed, 52 homohexameric molecular motors that harvest energy from ATP 53 to translocate substrates through their central pore (Jessop et al.,
54 2021). However, clamp loaders are not motors but instead are 55 ATP-dependent remodeling switches (Kelch, 2016). In contrast to 56 typical AAA+ ATPases, clamp loaders are heteropentameric with 57 the five different subunits called A-E (Figures 1A\&B). Each subunit 58 features a classic AAA+ ATPase module, which holds the ATP 59 sandwiched between the Rossmann fold and Lid domain at the 60 binding interface with the neighboring subunit. The AAA+ 61 modules of every subunit are extended by collar domains, which 62 tightly associate together into a flat disk, enabling dynamic 63 interactions between the five AAA+ modules.

64 In addition to the canonical $A A A+$ machinery, many clamp 65 loaders contain an A' domain that bridges the gap between the $A$ 66 and the $E$ subunit. The space between the $A^{\prime}$ domain and the $67 \mathrm{AAA}+$ domain of subunit $A$ is the "A-gate", (Figure $1 \mathrm{C}$ ), which 68 serves as the entry site for $p / t-D N A$ binding. It was initially 69 proposed that ATP binding triggers the five AAA+ modules to form 70 a spiral with a symmetrical pitch that matches the geometry of 71 DNA and templates the open clamp (Bowman et al., 2004; 72 Simonetta et al., 05/2009). This symmetric, helical arrangement of 73 the subunits results in a cracked interface between the A and $E$ 
A

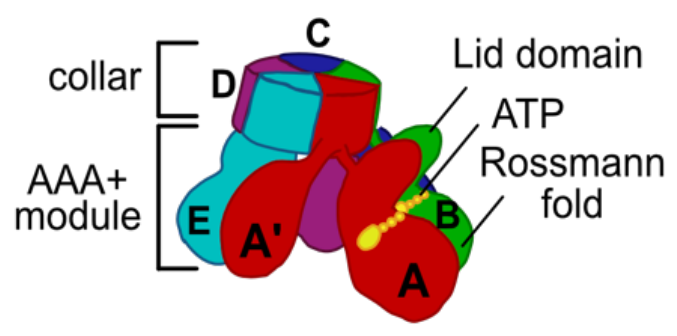

B

\begin{tabular}{|c|c|c|c|c|c|}
\hline \multicolumn{6}{|c|}{ A (RFC1) } \\
\hline$B R C$ & & ATPase & collar & $A^{\prime}$ & \\
\hline B (RFC4) & \multicolumn{5}{|c|}{ D (RFC2) } \\
\hline ATPase & |collar & & ATPase & & ollar \\
\hline C (RFC3) & \multicolumn{5}{|c|}{ E (RFC5) } \\
\hline ATPase & collar & & ATPase & & collar \\
\hline
\end{tabular}

C

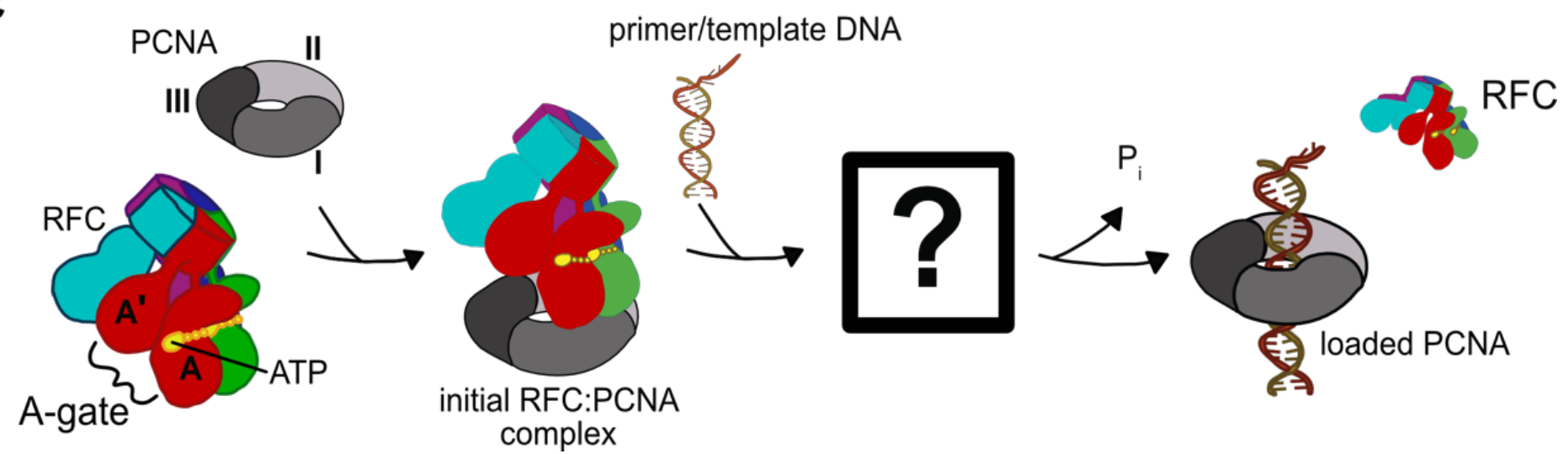

Figure 1. Architecture of the eukaryotic clamp loader (RFC) and clamp (PCNA). (A) RFC is composed of five different subunits (named $A$ to $E$ ) that each consist of the AAA+ ATPase module and a collar domain. The nucleotide binding site is sandwiched between the N-terminal Rossmann fold domain and the Lid domain of the ATPase module at the subunit interface. The ATPase module and a Cterminal extension of the A subunit called the A'-domain form the A-gate. (B) Domain organization of RFC subunits. (C) Clamp loading begins with binding of ATP to RFC, followed by PCNA binding. How PCNA is opened and DNA binds to the open RFC:PCNA complex is not known. DNA binding triggers ATP hydrolysis, PCNA closure, and RFC ejection.

74 subunits, bridged by the A' domain. As the A' domain stretches 75 away from the $A$ subunit to maintain contact, the A-gate opens 76 and permits p/t-DNA binding (Kelch et al., 2011). However, 77 structures of the human and yeast RFC:PCNA complexes bound 78 to ATP analog show a closed PCNA ring bound to RFC in an 79 autoinhibited state, where the closed A-gate blocks the DNA 80 binding (Bowman et al. 2004; Gaubitz et al. 2020). Additionally, 81 another element called the "E-plug" reaches into RFC's central 82 chamber and sterically occludes DNA binding. This autoinhibited 83 state of RFC bound to closed PCNA is likely the first intermediate 84 in the clamp loading reaction (Gaubitz et al., 2020b; Sakato et al., 85 02/2012; Thompson et al., 2012).

86 The question remains: How does the clamp loader open the 87 sliding clamp? This is perhaps the most important function of the 88 clamp loader, yet clues as to how this process is achieved remain 89 elusive (Figure 1C). The structure of the T4 phage loader bound to 90 DNA and an open clamp indicated that the clamp adopts a right91 handed spiral conformation that matches the helical pitch of DNA 92 (Kelch et al., 2011). However, this structure represents the state 93 after DNA is bound, and does not address how the clamp ring is 94 initially opened. Thus, the structure of a clamp loader bound to an 95 open clamp without DNA has been sought after, as it will 96 illuminate the opening process.
97 RESULTS

98 Structures of RFC:PCNA complexes en route to DNA loading

99 To understand how RFC opens PCNA and subsequently binds 100 DNA, we used single-particle cryo-EM to determine structures of 101 full-length $S$. cerevisiae RFC bound to PCNA and the slowly 102 hydrolyzing ATP analog ATPyS in the presence and absence of 103 primer-template (p/t) DNA. We reconstituted the complex from 104 purified RFC and PCNA subcomplexes that were separately 105 expressed in E. coli (Figure S1A). Full-length RFC is functional, as 106 it has the expected ATPase activity profile (McNally et al., 2010) 107 with PCNA and p/t-DNA synergistically activating ATP hydrolysis 108 (Figure 6F).

109 To prevent particle denaturation during sample preparation for 110 cryo-EM, we cross-linked DNA-free and DNA-bound complexes 111 using the amine-reactive crosslinker 112 bis(sulfosuccinimidyl)suberate (BS3). Mild cross-linking is 113 frequently used to obtain high-resolution cryo-EM structures of 114 labile complexes (Gerlach et al. 2018; Yoo et al. 2018; Gaubitz et 115 al. 2020). Mass spectrometry of the DNA-free sample reveals that 116 most cross-links are intra-molecular and map to the unresolved 


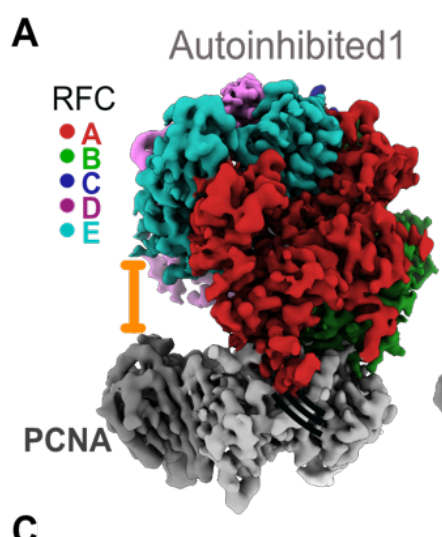

C

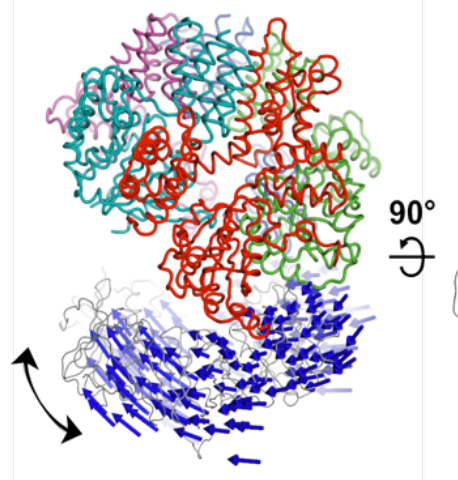

Autoinhibited2

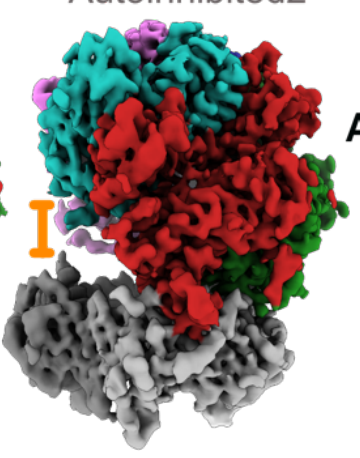

D
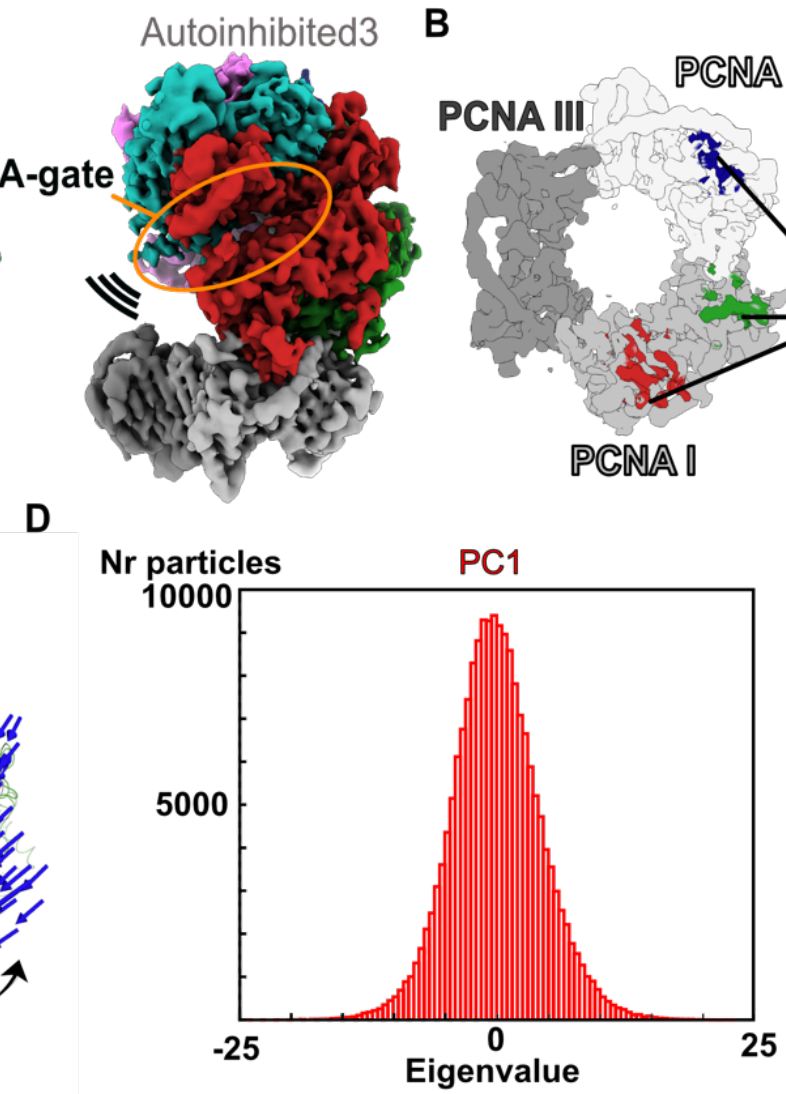

B

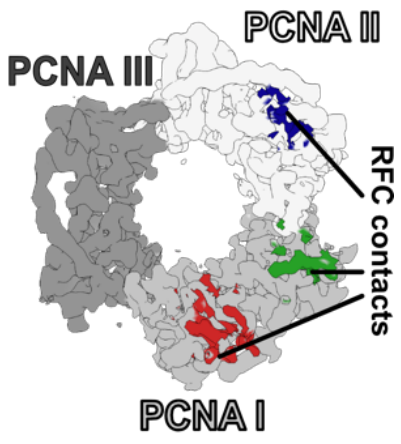

Eigenvalue

Figure 2. The Autoinhibited state is dynamic. (A) Cryo-EM maps of the three Autoinhibited conformations of the RFC:PCNA complex. PCNA tilts closer relative to RFC in Autoinhibited2. The subunit arrangement of the AAA+ module of Autoinhibited3 is changed slightly, which leads to a crack in the A-gate. (B) Top view on the contact sites of PCNA with RFC in the autoinhibited conformation. (C) Principal Component analysis of all Autoinhibited particles reveals a rocking motion of PCNA relative to RFC. The $\mathrm{C}_{\mathrm{a}}$ displacement of Principal Component 1 (PC1) is indicated by arrows, scaled down by a factor of 2. (D) Principal Component analysis reveals a range of motions within the initial RFC:PCNA complex. Amplitude histogram of the first principal component (PC1) reveals a unimodal distribution of particles, suggesting that this state consists of related particles in continuous motion.

$117 \mathrm{~N}$ - and C-termini of RFC1, with only a few detectable inter- 138 The initial complex of RFC:PCNA is dynamic

118 molecular cross-links between RFC subunits (Figure S1B; Table 139 Three of the classes from the DNA-free sample are of RFC bound 119 S1). No significant cross-links were observed between RFC and 140 to closed PCNA in different conformational states (Figure 2A). 120 PCNA.

141 Overall, these structures resemble the previous yeast RFC:PCNA

121 We imaged the RFC:PCNA complex with and without p/t-DNA 142 crystal structure and our recent cryo-EM structure of human RFC 122 using a 300 kV Titan Krios microscope (Figures S1C, S1D, S2A, 143 (hRFC):PCNA (Figure S4A; Bowman et al. 2004; Gaubitz et al. 123 S2B, S3A, S3B;Table S2). 3D classification results in four well- 144 2020). The PCNA ring is closed with only the A, B, and C subunits 124 defined reconstructions from the DNA-free sample, with overall 145 of RFC contacting PCNA (Figure 2B). The interaction area 125 resolutions ranging between 3.8 and $4.0 \AA$ (Figure S2D). The 146 between clamp loader and clamp averages $\sim 1940 \AA^{2}$ across the 126 dataset of the DNA-containing sample yielded several well 147 three states. The nucleotide density in each of the four active sites 127 defined classes, with overall resolutions ranging between 3.3 and 148 are most consistent with the presence of ATPYS, although the $1283.5 \AA$ (Figure S3C). We focused on classes in which all subunits 149 density for the $\gamma$-phosphate analog in the $D$ subunit is somewhat 129 of RFC and PCNA are visible, although the N- and C-terminal 150 ambiguous due to low local resolution throughout this subunit. 130 regions of the A subunit lack clear density. The quality of the cryo- 151 Nonetheless, the ATPase sites of the B, C, and D subunits are in 131 EM reconstructions readily permitted model building using the 152 an inactive state (Figure S4B), with the AAA+ spiral in the 132 crystal structure as a template (Bowman et al., 2004; Figures S1C, 153 overtwisted state observed in the hRFC structure and the 133 S1D; Table S2).

134

135

136

137 154 previous yeast RFC crystal structure (Figure S4C; Bowman et al. 155 2004; Gaubitz et al. 2020). Therefore, all three of these structures 156 represent autoinhibited states of RFC (termed Autoinhibited1, 

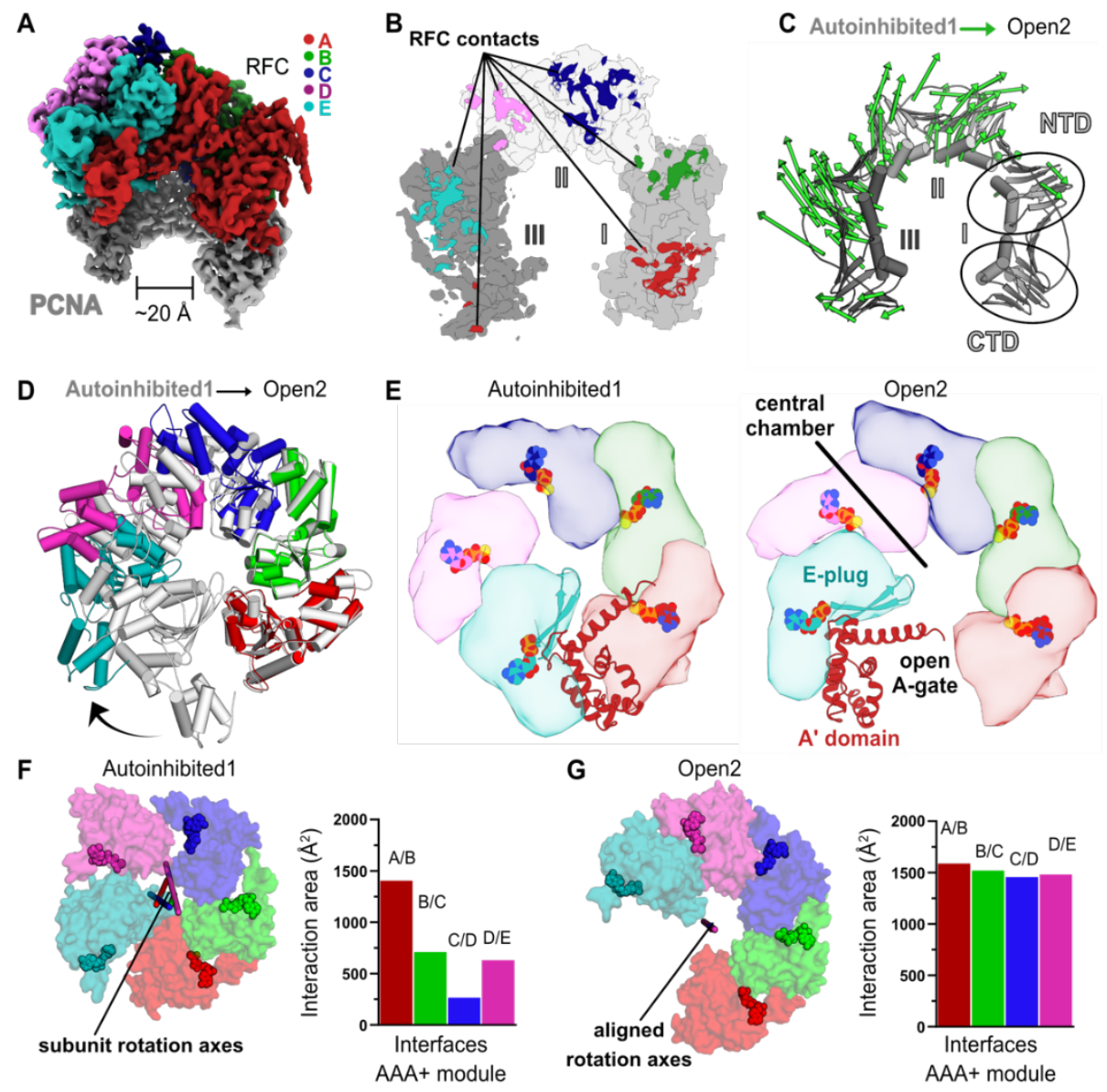

Figure 3: RFC undergoes a large conformational change to open PCNA. (A) Cryo-EM map of RFC bound to an open PCNA ring. (B) PCNA is held open by contacts with all five subunits of RFC. (C) The Ca displacement from closed to open PCNA is indicated by arrows, scaled up by a factor of four. (D) The AAA+ modules widen from the Autoinhibited state(gray) to an open spiral conformation. (E) Top view of the AAA+ spiral shows that the E-plug and A-gate block access to RFC's central DNA binding chamber in the Autoinhibited conformation but retract in the open conformation. RFC opens wide enough for DNA to directly enter the central chamber. (F) Top view of the Rossmann fold arrangement in the Autoinhibited conformation. The rotation axes that relate neighboring subunits are shown in different colors and are skewed, indicating asymmetric rotations which lead to gaps between the subunits. (G) The rotation axes overlay in the Open2 state of RFC, indicating a symmetric arrangement of the AAA+ spiral. Symmetrization closes the gaps, and results in an increased interaction area between neighboring subunits.

157 Autoinhibited2, and Autoinhibited3). Because the Autoinhibited1, 172

Despite these differences, the three Autoinhibited structures 158 2, and 3 states likely represent ATP-saturated configurations, we 173 are very similar, and so we asked if these conformations represent 159 place these conformational states early in the clamp loading 174 distinct intermediates or if they are snapshots along a continuum 160 reaction.

175 of conformations. Therefore we characterized the particles that 161 The subunits in the AAA+ spiral have a different tilt in each of 176 contribute to the Autoinhibited states using multi-body refinement 162 the Autoinhibited states, thereby slightly altering the intersubunit 177 (Figure S5), a computational tool that allows modeling of 163 interactions (Figure S4C). For instance, the Autoinhibited3 state 178 macromolecular motion (Nakane et al., 2018). To examine motion 164 exhibits a slightly cracked A-gate (but not open enough for DNA 179 between clamp and clamp loader, we defined RFC and PCNA as 165 to pass through), whereas the A-gate is closed in the 180 two independent rigid bodies (Figure S5A-S5D). This analysis 166 Autoinhibited 1 and 2 states (Figure 2A). Further, the AAA+181 revealed that the dominant motion is rocking of PCNA towards 167 modules of subunits C-D change their position into a more 182 RFC, with the linker between the ATPase and collar domains 168 symmetric alignment with overlapping rotation axes relative to 183 serving as a hinge (Figure 2C, Video S1). Other motions include 169 Autoinhibited1 and 2 (Figure S4C). On the other hand, the PCNA 184 swiveling of the RFC spiral with RFC-D getting closer to PCNA 170 ring tilts $\sim 19^{\circ}$ relative to the RFC-D in the Autoinhibited2 state 185 (Figure S5D, Video S2). These results are not dependent on the 171 relative to the Autoinhibited1 and 3 states (Figure 2A). 186 particular mask used, as similar motions are observed using 


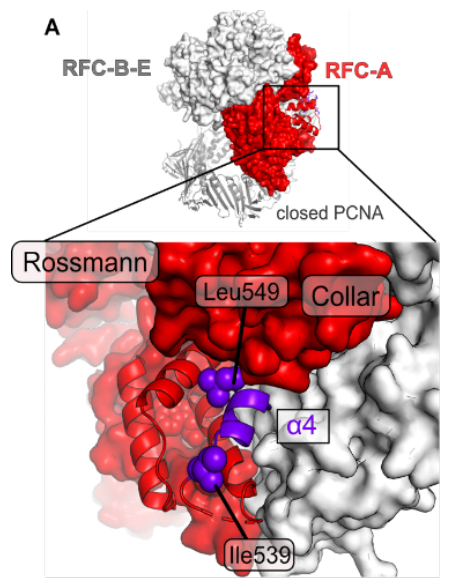

B

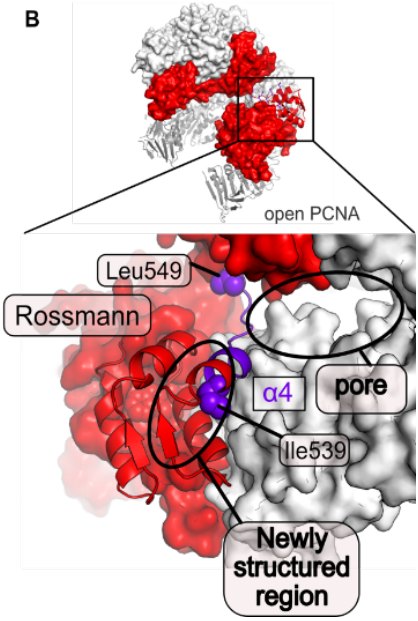

187 different masking strategies
(Figure

Figure 4: A fold-switch mechanism for opening a pore in the Open state of RFC:PCNA. (A) Helix 4 of the RFC-A subunit in 238 Autoinhibited1 is shown in purple. (B) In the open conformation, 239 Lid Helix 4 is displaced and partially unravels, whereby the 240 packing arrangement of the hydrophobic core of the lid domain in RFC-A changes. Ile536 and Leu549 move $\sim 13 \AA$ and $\sim 22 \AA$ from their original position and a pore is formed between the 243 RFC-A and RFC-B subunits.

188 component analysis of the multi-body conformers revealed
189 unimodal distribution of particles along their eigenvalue (Figures
190 2D, S5C, S5G). This unimodal distribution indicates that the thre
191 different observed cryo-EM class averages do not represent
192 particles in discrete states, but rather snapshots along
193 continuum of motion. Thus, the autoinhibited state of RFC
194 conformationally heterogeneous, with the dominant motio
195 driving RFC toward PCNA. We propose these motions represent
196 an early phase of the transition toward opening of the PCNA ring.

198 Each of the two cryo-EM datasets revealed a class of RFC bound 199 to open PCNA with no DNA bound (Figures S2D, S3C). To our 200 knowledge, these are the first high-resolution structures of a 201 clamp loader bound to an open clamp prior to DNA binding. Both 202 reconstructions are highly similar (overall $C_{a}$ RMSD is $0.74 \AA$, map 203 to map correlation coefficient is $\sim 0.85$, Figures S6A-S6D) and we 204 refer to these structures as Open1 and Open2. PCNA forms a 205 right-handed spiral with a $\sim 20 \AA$ opening that is wide enough for 263 206 dsDNA to enter (Figures 3A, B). The PCNA ring opens primarily 264 207 through in-plane rather than out-of-plane motions (in-plane $\sim 19 \AA 265$ 208 and out-of-plane $\sim 10 \AA$ for Open2, Figure 3C). Each of the 266 210 largest distortion in subunit II (Figure S6F).

211 PCNA opens at the A-gate of RFC, disrupting the interaction 269 212 between the first and third subunits of the PCNA ring (termed 270 213 PCNA-I and PCNA-III, hereafter). The open PCNA ring is directly 271 214 held by all five subunits of RFC, burying $\sim 3800 \AA^{2}$ of surface area, 272 215 an approximate $\sim 1860 \AA^{2}$ increase over that of the Autoinhibited 273 216 states (Figures 3B, S7A, S7B). The RFC-C subunit shifts 274 217 downward to interact much more tightly with PCNA-II, while 275 218 PCNA also forms new interactions with RFC-D, RFC-E, and the 276

219 A' domain of RFC-A (Figures S7C, D). The overall interface is 220 characterized by an alternating pattern of strong and weak 221 interactions (strong: RFC-A, -C, \& -E; weak: RFC-B, -D, \& A'). The 222 strong interactions are with the main partner binding pocket of 223 PCNA, using a binding region that resembles a common motif for 224 PCNA-interacting partners. Of these strong interfaces, RFC-A is 225 the most substantial and RFC-E weakest; RFC-A contains a true 226 PCNA interaction motif, while RFC-C and RFC-E's motifs are 227 increasingly degenerate. It is likely that the stronger interactions 228 at the "bottom" of the spiral allows the clamp loader to toggle 229 between the closed and open states of PCNA without releasing 230 RFC.

231 The $\mathrm{AAA}+$ modules of RFC adopt a right-handed spiral whose 232 periodicity matches that of the six contact sites on PCNA. The 233 symmetry of the ATPase spiral can be visualized by the near 234 perfect alignment of the rotation axes that relate adjacent AAA+ 235 subunits (Figures 3F-G). The interfaces between adjacent AAA+ 236 modules also become tighter, bringing the catalytic arginine finger 237 residue closer to the neighboring ATPase site. This observation 38 explains the modest boost in ATP activity upon PCNA binding 39 (Johnson et al., 2006; Figure 6F). Similar to the Autoinhibited 240 structures, all four active sites are bound to ATP analog (Figure 241 S6E). Therefore, ATP hydrolysis is not necessary to drive the 242 conformational change from Autoinhibited to Open.

243 In order to rupture the PCNA ring, the AAA+ spiral of RFC 244 widens, opening the A-gate. RFC opens using a large hinge 245 motion, pivoting around the $B-C$ and $C-D$ subunit interfaces 246 (Figure 3D, 3E; Video S3). The RFC-E subunit uses its E-plug to 247 bind PCNA, which pulls the A' domain and E-plug up to $45 \AA$ away 248 from the $A A A+$ module. This reveals a large opening of the A-gate 249 (at its most narrow, the A-gate is approximately $20 \AA$ wide) (Figure $2503 \mathrm{E})$. p/t-DNA can therefore directly enter the open RFC:PCNA 251 complex.

252 Opening of the A-gate separates the RFC-A Lid and collar 253 domains, inducing a fold-switching transition in the Lid domain. 254 The majority of the last helix of the Lid (Helix a4; residues 541255546 ) unravels into a taut $\beta$-strand conformation (Figures $4 \mathrm{~A}, 4 \mathrm{~B}$ ). 256 The remaining residues in helix a4 (residues 536-542) shift 257 forward, causing a major change in the core packing of the Lid 258 domain. This "sliding spring" motion leads to a $\sim 11 \AA$ helix 259 displacement, whereby some residues, such as Leu 549, move up 260 to $22 \AA$ from their original position. The stretching of the RFC-A 261 Lid opens a new pore between the $A$ and $B$ subunits (Figure 4B). 262 We discuss the role of this pore in the next section.

\section{Structures of the RFC:PCNA complex bound to primer-} 64 template DNA

65 To reveal how RFC:PCNA binds and responds to DNA, we 66 analyzed two classes that contain DNA-bound RFC:PCNA. One 267 class shows PCNA in an open lock-washer shape, and the other 268 has PCNA in a closed conformation. Therefore, we term these two 69 states DNA ${ }^{\text {PCNA-open }}$ and DNA ${ }^{\text {PCNA-closed }}$, respectively (Figures 5A$705 \mathrm{C})$. Both classes contain clear density for $\mathrm{p} / \mathrm{t}-\mathrm{DNA}$ : 18 base pairs 71 of duplex DNA are bound inside the central chambers of RFC and 2 PCNA, and 6 nucleotides of the ssDNA template extend through 3 the A-gate, preventing its closure. The AAA+ spiral of RFC tracks 74 the minor groove of dsDNA using a suite of residues that are 75 conserved across all known clamp loaders to match the helical 6 symmetry of DNA (Kelch et al., 2011; Simonetta et al., 05/2009). 


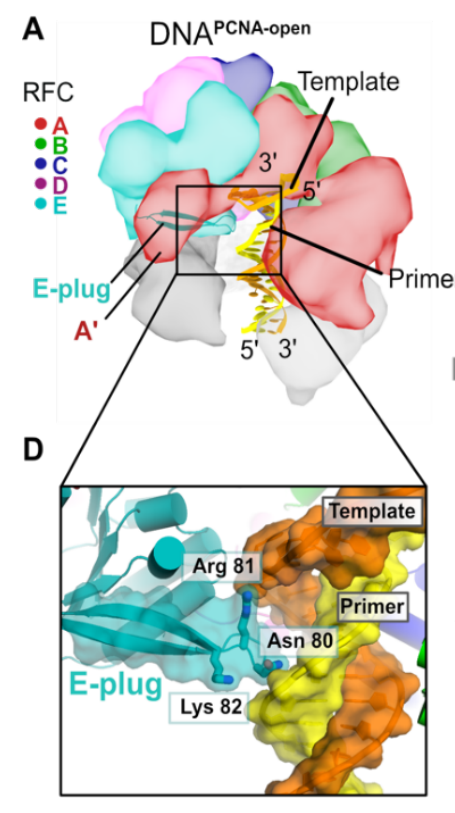

G

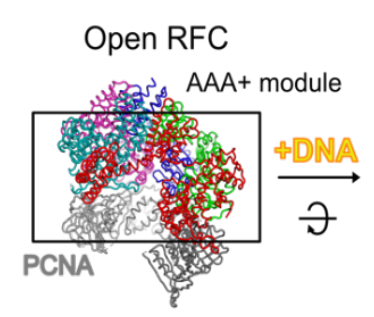

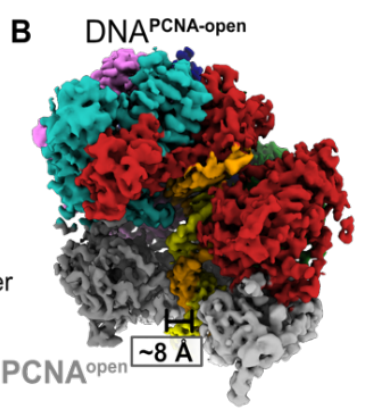

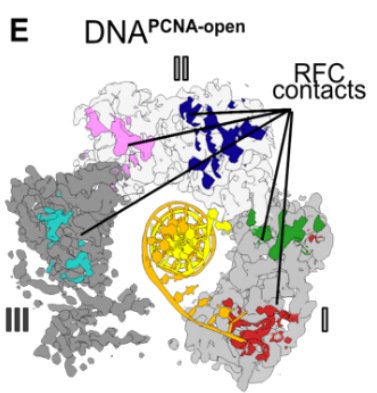

H

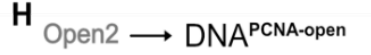

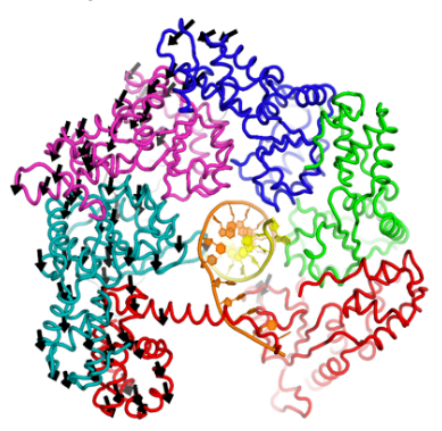

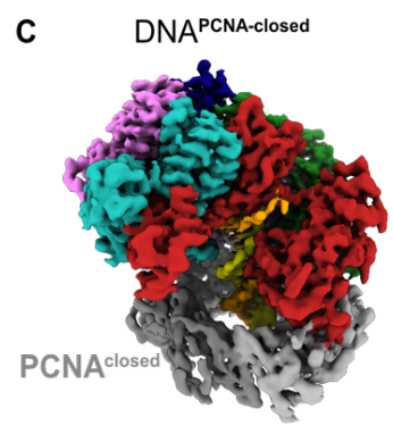

$\mathbf{F}$ DNA PCNA-closed $^{\text {Pan }}$

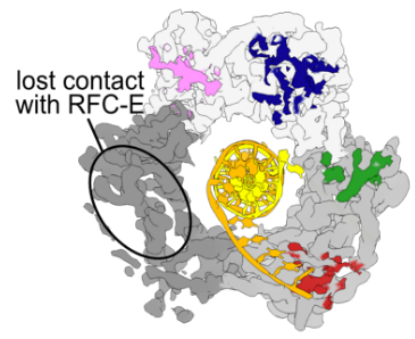

$I_{\text {DNAPCNA-open }} \longrightarrow$ DNAPCNA-closed

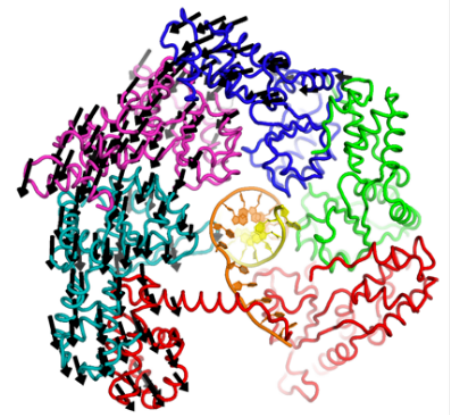

Figure 5: Structures of RFC:PCNA bound to DNA. (A) Schematic representation of the structure of RFC:PCNA bound to primer-

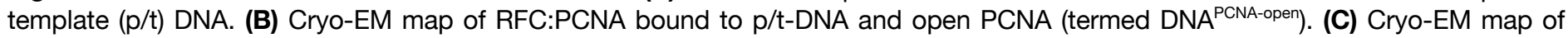

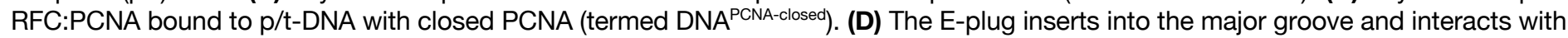
both strands of the p/t-DNA. (E) Top view of contact sites of RFC with PCNA. PCNA is held open by contacts with all five subunits in

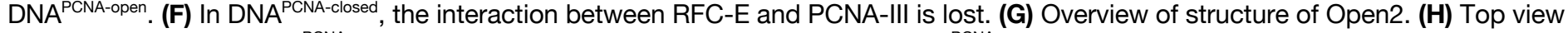
of the AAA+ spiral of DNA ${ }^{\mathrm{PCNA}-\text { open }}$. Displacement vectors between Open2 and DNA ${ }^{\mathrm{PCNA}-\text { open }}$ are indicated by arrows, scaled up by a factor of two. The AAA+ spiral constricts around DNA. (I) The AAA+ spiral of DNA ${ }^{\text {PCNA-closed }}$. Displacement vectors between DNA ${ }^{\text {PCNA-open }}$ and DNA ${ }^{\text {PCNA-closed }}$ indicate that the AAA+ spiral constricts further around DNA, leading to changes in ATPase sites.

277 The E-plug beta-hairpin slots into the major groove of the 289 bind DNA with equivalent affinity as WT-hRFC (Gaubitz et al., 278 duplex region of $\mathrm{p} / \mathrm{t}-\mathrm{DNA}$ (Figure 5D). Conserved basic residues 290 2020b).

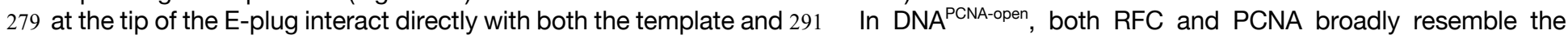
280 primer strands. Therefore, the E-plug provides a mechanism for 292 conformations seen in Open1 and Open2. The RFC A-gate is 281 the RFC AAA+ spiral to recognize both strands of DNA, unlike the 293 open, with all 5 subunits gripping PCNA in an open lock-washer 282 clamp loaders from E. coli and T4 phage, whose AAA+spirals only 294 shape. However, both RFC and PCNA constrict relative to the 283 interact with the template strand (Kelch et al., 2011; Simonetta et 295 Open1 and Open2 structures (Figures 5G, 5H and Figure S8A). 284 al., 05/2009). Moreover, this structure shows that the E-plug 296 RFC constricts modestly, pivoting the E, D, and C subunits 285 changes its role from blocking DNA binding (in the three 297 around a hinge at the B-C interface. PCNA constricts $\sim 12 \AA$ upon 286 Autoinhibited states) to one in which it directly supports DNA 298 DNA binding, with most of this constriction occurring in subunit III 287 binding. This explains the non-intuitive effect on DNA binding we 299 of PCNA (Figure S8A). Subunit III of PCNA is held by the RFC-D 288 observed previously, where hRFC variants with a mutated E-plug 300 and RFC-E subunits, although RFC-E grips PCNA less tightly in 301 DNA $^{\text {PCNA-open }}\left(\sim 3800 \AA^{2}\right.$ total RFC-PCNA interaction area for the 
A unpaired 3' end
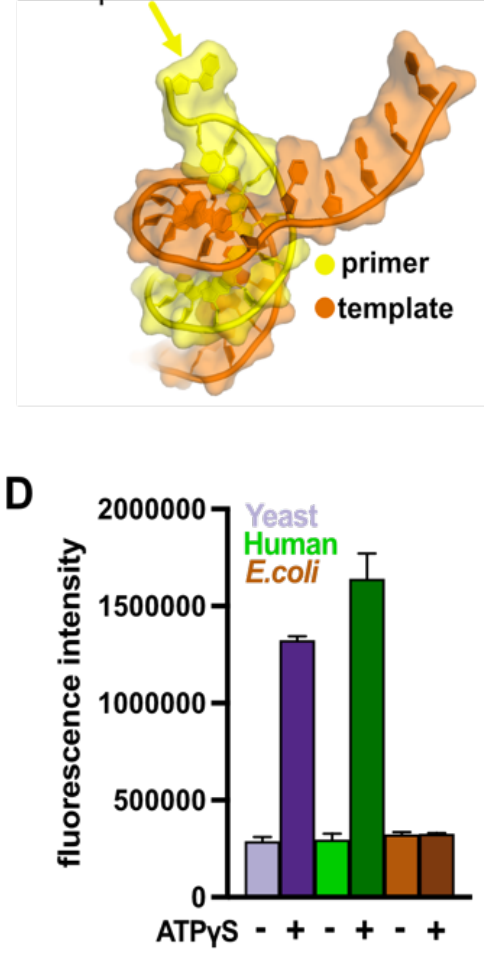

B

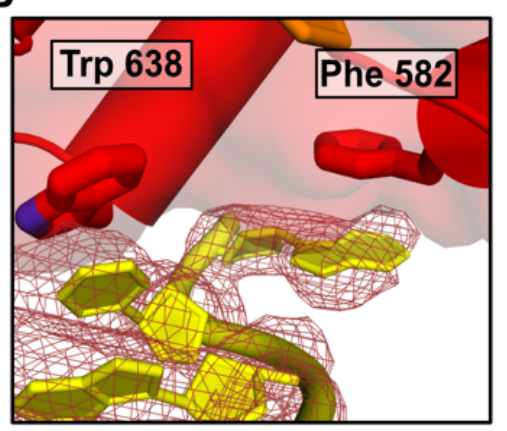

$\mathrm{E}$

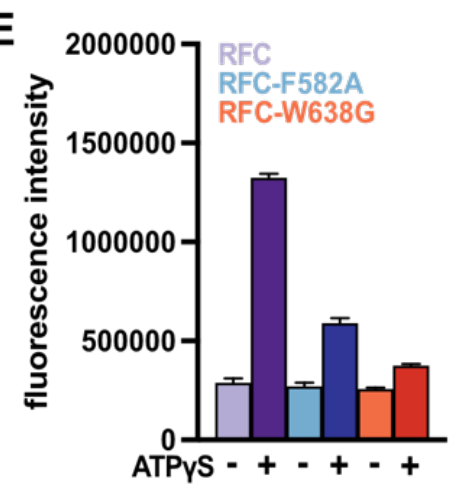

C

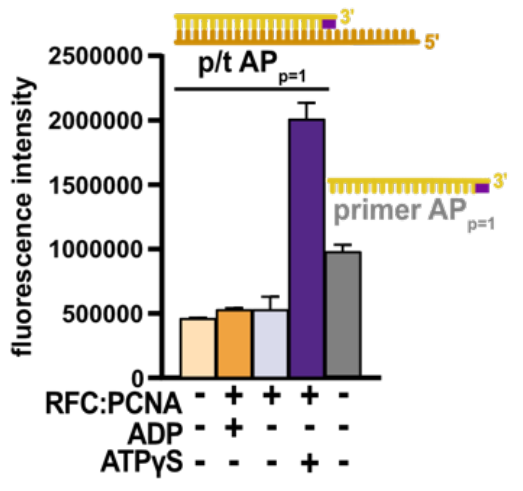

$\mathbf{F}$

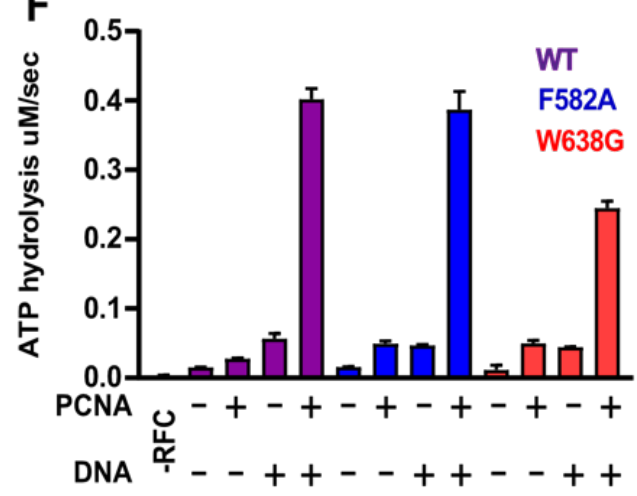

Figure 6: RFC separates the 3' end of the primer strand. (A) The last nucleotide in the primer strand is separated from the duplex. (B) The collar of RFC-A contains a "separation pin" with two critical residues (Trp638 and Phe582) that stabilize the flipping of the 3' primer nucleotide into the pore between RFC-A and RFC-B. The cryo-EM map is shown in red mesh. (C) The primer strand of p/t-DNA contains 3' nucleotide with a 2-aminopurine (2AP) base, an adenine analog that reports on base-pairing and base-stacking. 2AP fluorescence increases in the presence of ATPYS and RFC:PCNA to a higher level than in the unpaired 2-aminopurine-labeled primer strand. (D) The human RFC:PCNA complex also induces an increase in 2AP fluorescence emission, whereas the $E$. coli clamp loader, which does not flip the $3^{\prime}$ ' end of the primer (Simonetta et al., 05/2009), does not increase 2AP fluorescence. (E) Mutation of Phe582 and Trp638 reduces 2-AP fluorescence in the presence of ATPyS. (F) ATPase activity of the "separation pin" mutants.

302 Open1\&2 structures vs $\sim 3400 \AA^{2}$ for DNA ${ }^{\text {PCNA-open }}$. Overall, the 323 subunit (Figure S8C). Therefore, these structures represent 303 PCNA conformation is similar to that seen for the structure of the 324 reaction intermediates following DNA binding but preceding ATP 304 T4 phage clamp bound to clamp loader and p/t-DNA (Kelch et al., 325 hydrolysis. Upon binding DNA, the AAA+ spiral constricts (Figure 305 2011).

$3265 \mathrm{H}$ ), primarily due to a hinge-like motion at the interface between 306 The DNA ${ }^{\text {PCNA-closed }}$ structure has a closed PCNA ring that is 327 RFC-C and RFC-B. The AAA+ spiral constricts around an axis 307 distorted from planarity. Upon closure, PCNA loses its interaction 328 coincident with the DNA axis. Subsequent PCNA closure further 308 with the RFC-E subunit, but retains its interfaces with the other 329 exaggerates the constriction of the RFC AAA+ spiral (Figure 5I). 309 four RFC subunits (Figures 5E, 5F). The distortion of the PCNA 330 This motion brings adjacent AAA+ modules into close contact, 310 ring is most prevalent in subunit III, which puckers to maintain its 331 enabling the active sites to fully assemble. The close apposition 311 interaction with the RFC-D subunit (Figure S8A). Interestingly, the 332 of neighboring subunits brings each of the arginine finger residues 312 interaction between DNA and PCNA becomes more extensive 333 in close contact with the gamma-phosphate of ATP, which is 313 upon PCNA closure ( 50 $\AA^{2}$ vs $\left.250 \AA^{2}\right)$. Conserved basic residues 334 necessary for catalytic activity (Johnson et al., 2006). However, a 314 lining the inner pore of PCNA also interact directly with the duplex, 335 conserved arginine known as the switch residue is not in the 315 as has been hypothesized previously (Liu et al., 2017; McNally et 336 position that was previously predicted to be required for activation 316 al., 2010). We propose that these interactions help to drive the 337 of the Walker B glutamate (Kelch et al., 2011, 2012). Despite this, 317 closure of PCNA around DNA.

338 the active sites appear to be in the fully active state, with all of the 318 DNA binding and PCNA closure also induce constriction of the 339 catalytic machinery poised to hydrolyze ATP. We discuss the 319 RFC AAA+ spiral, potentiating ATPase activity. DNA ${ }^{\text {PCNA-open }}$ and 340 ramifications of this observation on the allosteric activation of RFC $320 \mathrm{DNA}^{\mathrm{PCNA}-\text {-closed, }}$, just like the other states described herein, are in the 341 below.

321 fully ATP-bound state: ATP $\gamma S$ in the active sites of the A, B, C, 342

322 and D subunits, and ADP in the nucleotide-binding site of the E 343 RFC flips the $\mathbf{3}^{\prime}$ base of the primer strand 
344 Unexpectedly, we observe that the $3^{\prime}$ nucleotide of the primer 402 state in which PCNA is opened but DNA has yet to bind. The

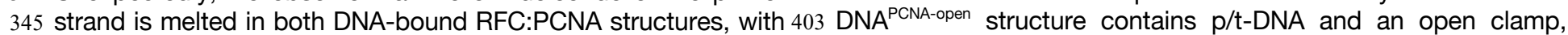
346 the base flipped away from the rest of the duplex (Figure 6A, 6B). 404 which is the transient intermediate following DNA binding (Liu et 347 The base pair is disrupted by a "separation pin" at the base of the 405 al., 2017; Marzahn et al., 2014; Sakato et al., 02/2012). Finally, the 348 RFC-A collar domain that wedges between the DNA strands 406 DNA ${ }^{\text {PCNA-closed }}$ structure represents the stable intermediate that 349 (Figure 6B). The indole ring of Trp638 replaces the flipped 3' base 407 forms prior to ATP hydrolysis (Marzahn et al., 2014; Sakato et al., 350 to maintain stacking interactions. The $3^{\prime}$ nucleotide is 408 2012). Therefore, our structures delineate the conformational 351 repositioned inside the pore formed by the unraveling of the RFC- 409 states that span the entire clamp opening and closing process, 352 A Lid domain upon opening of the A-gate; this site is closed in the 410 the central reaction of the clamp loading cycle.

353 Autoinhibited state (Figure 4). The flipped base stacks against the

354 phenyl ring of Phe582. These residues are conserved in 411 A crab-claw mechanism for opening the sliding clamp

355 eukaryotic clamp loaders but are absent in bacterial, archaeal or 412 Our structures show that RFC is in a constricted, auto-inhibited

356 phage clamp loaders (Figure S9 E). 358 substrates containing the adenine analog 2-aminopurine (2AP). 415 heterogeneity using multi-body refinement. The primary mode of 359 Fluorescence of 2AP is dependent on base-pairing (Frey et al., 416 motion pivots PCNA relative to RFC, such that PCNA approaches 360 1995; Jean and Hall, 2001): fluorescence is low when 2AP is base 417 the D- and E-subunits of RFC. We speculate that this motion is 361 paired, but high in the free state. To monitor melting, we placed 418 on-pathway toward a direct interaction between PCNA and all five 362 2AP either at the $3^{\prime}$ end of the primer strand $\left(\mathrm{AP}_{\mathrm{p}=1}\right)$ or at the 419 RFC subunits, facilitating the opening of the PCNA ring. Thus, the 363 corresponding site in the template strand $\left(\mathrm{AP}_{\mathrm{t}=1)}\right.$. Importantly, we 420 dynamics of the Autoinhibited complex are important for the 364 find a dramatic increase in 2AP fluorescence that is dependent on 421 opening of PCNA. Future studies will investigate this possibility. 365 addition of RFC, PCNA and ATP analog (Figures 6C, S9A-C). The 422 To open PCNA, our structures show that RFC uses the 366 increase in 2AP fluorescence is not observed in the presence of 423 previously hypothesized "crab-claw" mechanism (Jeruzalmi et al., 367 ADP, which does not support DNA binding (Kelch et al., 2011; 424 2001a, 2001b; O'Donnell et al.). This contradicts the previous 368 McNally et al., 2010). Placement of $2 A P$ at the $\mathrm{p}=2$ or $\mathrm{p}=3$ position 425 suggestion that the $E$. coli clamp loader opens the ring with limited 369 of the primer yields diminished fluorescence, suggesting that only 426 conformational changes in the clamp loader (Goedken et al., 370 the $3^{\prime}$ base is flipped (Figure S9B). Therefore, our 2AP 427 2004; Kelch, 2016). In this "limited change" model, ATP binding 371 experiments validate that RFC- and PCNA-dependent $3^{\prime}$ end 428 places the encounter complex in a conformation that "templates" 372 melting occurs in solution. The human clamp loader, which has a 429 the open clamp. However, our structures preclude this model for 373 similar separation pin as yeast RFC (Gaubitz et al., 2020b), greatly 430 RFC because we observe large conformational changes in the 374 enhances 2AP fluorescence. However, the E. coli clamp loader, 431 clamp loader upon opening the PCNA ring. Furthermore, the 375 which binds $\mathrm{p} / \mathrm{t}-\mathrm{DNA}$ but does not melt the primer strand 432 Autoinhibited state of RFC can not template an open PCNA 376 (Simonetta et al., 05/2009), does not alter fluorescence (Figure 433 conformation. One possible reason for the discrepancy between 377 6D). Thus melting of the $3^{\prime}$ nucleotide is a conserved activity of 434 the two studies is that different model systems were used; 378 eukaryotic clamp loaders, but is likely not used by bacterial clamp 435 bacterial clamp loaders lack the $A^{\prime}$ domain that constricts the 379 loaders.

380 To determine the mechanism and role of primer melting, we 437 bacterial clamp loaders may be free to adopt a conformation that 381 modified the $\mathrm{p} / \mathrm{t}-\mathrm{DNA}$ and/or key residues in the separation pin 438 can template the open clamp prior to clamp binding.

382 and assessed their effects on base flipping, ATP hydrolysis, and 439 The crab-claw motion that we observe is primarily driven by a 383 DNA affinity. The W638G and F582A variants have attenuated 440 hinge-like motion that pivots about the RFC-C subunit. This 384 base-flipping as measured by 2AP fluorescence (Figures 6E, 441 motion allows the A' domain and E, D, and C subunits to grip 385 S9C). However, DNA-dependent ATP hydrolysis is minimally 442 PCNA tightly, which is impossible in the Autoinhibited state. 386 affected, particularly in the F582A variant, whose ATPase rate and 443 Kinetic characterization of RFC variants has predicted a hinge role 387 apparent affinity for DNA are unchanged from WT (Figures 6F and 444 for this region (Sakato et al., 2012), highlighting this subunit's 388 S9D). These results indicate that base flipping requires the 445 importance in clamp loading. The crab-claw conformational 389 "separation pin", but base flipping is not required for DNA binding 446 change is remarkable because it requires a fold-switching event 390 or ATPase activation.

391

447 in the Lid domain of the RFC-A subunit (Figure 4A, 4B). At a 448 minimum, this would require that helix-4 of the RFC-A Lid to 449 unfold and refold into a new position. The fact that clamp opening

\section{DISCUSSION}

\section{Defining the clamp loading reaction in high-resolution}

394 We have determined a series of structures that provide a high395 resolution view of the clamp loading process. Our structures 396 correspond to numerous reaction intermediates, allowing us to 397 order the structures into a coherent description of the clamp 398 loading reaction prior to ATP hydrolysis (Figure 7 and Video S4). 399 The Autoinhibited states represent the transient encounter 400 complex that forms early in the clamp loading process before ring 401 opening. The Open $1 \& 2$ states represent the stable intermediate 450 is relatively fast (Liu et al., 2017) and does not require ATP 451 hydrolysis indicates that these conformational rearrangements 452 must have a relatively low energy barrier despite the large-scale 453 motion. How the RFC:PCNA complex couples these motions 454 becomes an important question for future studies.

455 Why use a "crab-claw" mechanism? We envision two non456 mutually exclusive hypotheses. First, we hypothesize that this 457 mechanism allows RFC to bind each of its macromolecular 458 substrates (PCNA and $p / t-D N A$ ) in the proper order to ensure 459 efficient clamp loading and to avoid futile cycles of ATP 


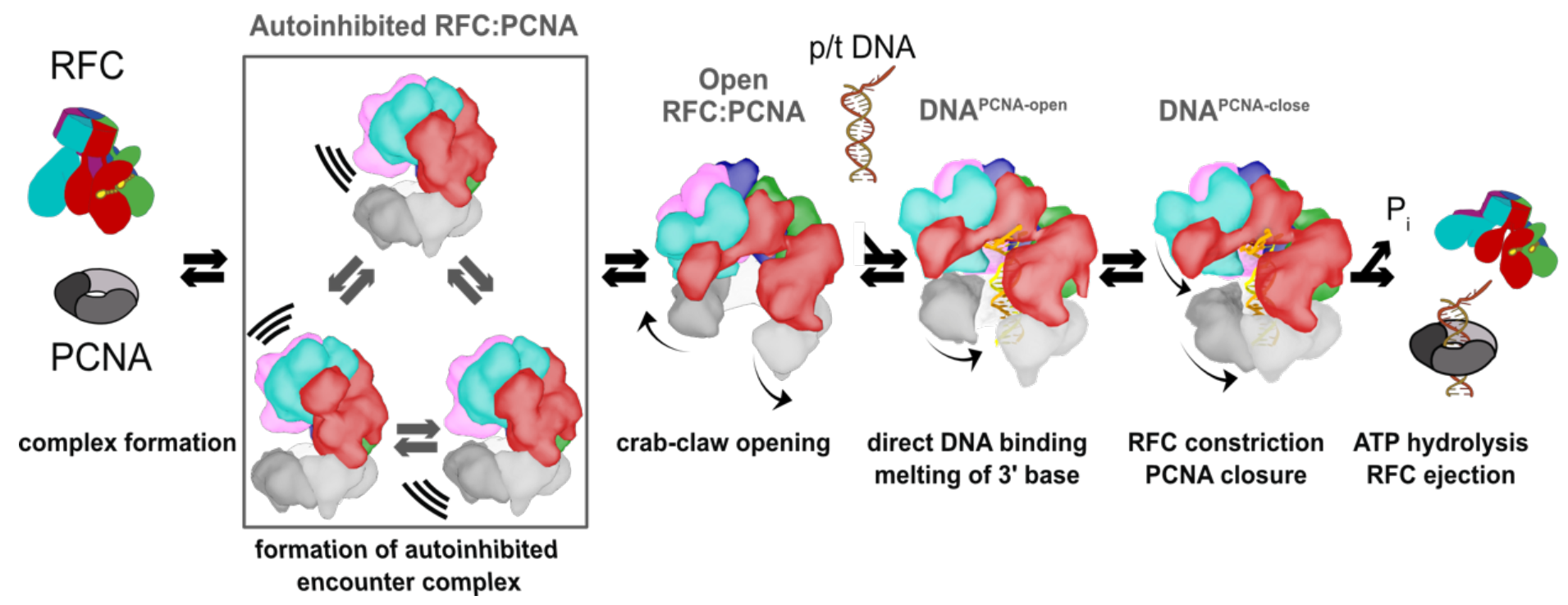

Figure 7: Clamp loading by RFC. Initial binding of RFC to PCNA places the complex in an Autoinhibited state, whereby closed PCNA and the E-plug preclude DNA binding, and an overtightened AAA+ helix inhibits ATPase activity. The Autoinhibited state is dynamic, rocking PCNA relative to RFC as captured by multibody refinement. Upon complete binding to PCNA, RFC uses the crab-claw mechanism to simultaneously open both PCNA and the A-gate, providing an entryway for p/t-DNA. p/t-DNA then binds directly through the A-gate and open PCNA, which are wide enough to accommodate dsDNA entry. The 3' end of the primer is flipped into the pore that is formed between RFC-A and RFC-B. PCNA closes to form additional contacts with DNA, partially detaching from RFC at the $E$ subunit. Finally, ATPase activity and inorganic phosphate release eject RFC, leaving PCNA bound to p/t-DNA in the correct orientation.

460 hydrolysis. For proper clamp loading, RFC must bind PCNA first, 492 into the AAA+ spiral of the T4 and E. coli clamp loaders than in 461 because initial binding of $p / t-D N A$ would sterically hinder binding 493 RFC (Figure S10A). Therefore, the large swing in the proportional 462 of the PCNA ring. Therefore, RFC has evolved high affinity for 494 interaction area is the net result of additional interactions from 463 PCNA and only binds p/t-DNA with high affinity after it has bound 495 RFC-A and less from the remaining subunits.

464 PCNA (Cai et al., 1998; Shiomi et al., 2000). The crab-claw 496 This proportionally large interaction area suggests RFC-A as 465 mechanism for PCNA opening can explain this hierarchy of 497 the subunit primarily responsible for recognizing DNA. This finding 466 binding, as the auto-inhibited state blocks the DNA binding site 498 provides an attractive explanation for how alternative clamp 467 ((Gaubitz et al., 2020b) and Figure 3E). The crab-claw mechanism 499 loaders specifically recognize different DNA structures. RFC-like 468 ensures that RFC's DNA-binding chamber only becomes 500 Complexes or RLCs are only found in eukaryotes and share four 469 accessible once the PCNA ring is open. Our second hypothesis is 501 of RFC's five subunits (RFC-B through RFC-E); each RLC 470 that the crab-claw mechanism enables complex modes of clamp 502 contains a unique A subunit (Majka and Burgers, 2004). We 471 loader regulation. Clamp loader activity could be inhibited by 503 hypothesize that the diminished role of the B, C, D, and E subunits 472 binding partners or post-translational modifications that favor the 504 in DNA recognition allows the A-subunit to assume the role of 473 Autoinhibited state. There are numerous RFC binding partners 505 specifically binding unique structures of DNA. In support of this 474 and post-translational modifications that remain unexplored, and 506 hypothesis, bacterial and phage clamp loaders do not have 475 thus are candidates for playing regulatory roles (Dephoure et al., 507 alternative forms that recognize different DNA structures, and 476 2008; Kim and Brill, 2001; Ochoa et al., 2020; Olsen et al., 2010; 508 their clamp loaders have substantially more contact between DNA 477 Tomida et al., 2008; Wang et al., 2012, 2013).

478

479 RFC-A subunit drives DNA recognition

509 and the $B, C, D$, and $E$ subunits. The more pronounced role of the 510 A subunit in eukaryotic clamp loaders allows for dramatically more 511 plasticity in function. Further, the diminished role of the remaining 480 To illuminate how RFC recognizes DNA, we measured the relative 512 subunits raises the question of how the pivot point at the C 481 contribution of each RFC subunit to DNA binding. We find that 513 subunit contributes to the activity of RLC complexes. Finally, 482 RFC-A accounts for $\sim 64 \%$ of the buried surface area between 514 these findings raise the intriguing possibility of engineering RLCs 483 RFC and DNA. This contrasts with T4 and E. coli clamp loaders, 515 with novel specificity and activity.

484 where the A subunits account for $\sim 36 \%$ of the binding interface 516 Following this reasoning, we hypothesize that RFC flips the $3^{\prime}$ 485 (Figure $\mathrm{S} 1 \mathrm{OB}$ ). Much of this proportional increase arises from 517 nucleotide to specifically recognize the recessed $3^{\prime}$ end of $\mathrm{p} / \mathrm{t}$ 486 additional interactions between RFC-A and DNA through the 518 DNA. We observe flipping of the $3^{\prime}$ nucleotide in both the DNAPCNA487 separation pin and the flipped $3^{\prime}$ nucleotide. Furthermore, we find $519{ }^{\text {open }}$ and DNA ${ }^{\text {PCNA-closed }}$ structures, indicating that flipping occurs 488 that $B, C, D$, and E subunits of RFC interact with DNA significantly 520 before ring closure. This observation can explain the "DNA 489 less $\left(\sim 760 \AA^{2}\right)$ than the comparable subunits of T4 and $E$. coli 521 repositioning transition" that occurs quickly (t1/2 35 ms) after $490\left(\sim 1125 \AA^{2}\right)$. The decrease in DNA interaction from the B, C, D, and 522 initial DNA binding, but before clamp closure (Liu et al., 2017). We $491 \mathrm{E}$ subunits is due to the $\mathrm{p} / \mathrm{t}-\mathrm{DNA}$ duplex region inserting deeper 
523 propose that this transition is the flipping of the $3^{\prime}$ nucleotide. 583 and p/t-DNA. These tight interactions are critical because much 524 Future experiments will investigate this possibility in more detail. 584 of the catalytic machinery for ATP hydrolysis is provided in trans 525

\section{Forces driving clamp loading} 585 by neighboring subunits (Johnson et al., 2006). However, two 586 observations stand out as surprising. First, the $A A A+$ spiral is 527 Our structures delineate a conformational pathway that illustrates 587 actually less symmetric once p/t-DNA binds (Figure S8B). This 528 much of the clamp loading reaction. We reveal how: 1) RFC 588 was unexpected because DNA had been thought to be the driving 529 initially binds PCNA, 2) how PCNA is opened, 3) how DNA is 589 force for symmetrizing the AAA+ spiral (Kelch et al., 2011 ; 530 bound, and 4) how PCNA closes around DNA. This 590 Simonetta et al., 05/2009), and this symmetry had been thought 531 unprecedented view into the mechanism of clamp loading allows 591 to favor ATP hydrolysis. But the RFC:PCNA complex 532 us to hypothesize on the forces that drive this reaction towards 592 (corresponding to the the Open1 and Open2 structures) has $\sim 5-$ 533 the loading of PCNA. We use the interaction areas between and 593 10-fold lower ATPase activity than when both PCNA and DNA are 534 within PCNA, RFC, and p/t-DNA to approximate these forces. 594 bound (Figure 6F, Chen et al., 5/2009; Gomes et al., 2001; 535 PCNA is opened through a large conformational change in both 595 McNally et al., 2010; Sakato et al., 02/2012). The second 536 PCNA and RFC. In solution the open form is the predominant 596 surprising observation is that a set of conserved arginines (termed 537 state (Zhuang et al., 2006), so it is important to understand what 597 the arginine switch residues) within the core of the AAA+ module 538 interactions drive this opening. Upon opening, PCNA loses the 598 are not in direct contact with DNA. The arginine switch residues 539 entire interface between subunits I and III. However, the open 599 had been hypothesized to flip outward to grip DNA, thereby 540 PCNA ring increases its interaction area with RFC by contacting 600 releasing the catalytic glutamate to activate ATP hydrolysis (Kelch 541 all five subunits. Moreover, the crab claw motion of RFC results in 601 et al., 2011). The lack of flipping of the arginine switch residues in 542 tighter association between adjacent AAA+ modules. Altogether, 602 response to DNA binding argues that the proposed arginine 543 the opening of PCNA and RFC result in an increased interaction 603 switch mechanism is not critical for sensing and responding to 544 area of $\sim 4000 \AA^{2}$ (Figure 3F). We propose that this is the driving 604 DNA binding. Our observations are in agreement with previous 545 force for stabilizing the open form of PCNA.

605 studies that found that the arginine switch residues of RFC do not 546 Once open, p/t-DNA enters the PCNA:RFC complex through 606 likely play a direct role in activating ATP hydrolysis, but are 547 the A-gate. The A-gate is wide enough for dsDNA to directly enter 607 important for the synergistic activation by both PCNA binding and 548 into the RFC:PCNA inner chamber. This finding is in direct 608 DNA binding (Liu et al., 2017). Further studies will be necessary to 549 contradiction of the "filter-and-slide" model for DNA binding that 609 reveal how RFC integrates binding of both PCNA and p/t-DNA to 550 posited that the opening is large enough for only ssDNA to enter 610 achieve full activation.

551 such that the clamp loader filtered out dsDNA to accelerate the 611

552 search for a p/t-junction (Kelch, 2016; Kelch et al., 2011, 2012). 612 Comparison with other AAA+ machines

553 The filter-and-slide model was primarily predicated on crystal 613 Clamp loaders have long been models for structure and 554 structures of the T4 phage clamp loader and on FRET data that 614 mechanism of AAA+ proteins (Guenther et al., 1997). However, 555 suggested that initial binding of DNA does not constrict the open 615 they are unusual in that they are pentameric protein remodeling 556 clamp (Kelch et al., 2011; Zhuang et al., 2006). While it remains a 616 switches instead of the more typical hexameric rings that act as 557 possibility that other clamp loaders use a filter-and-slide 617 processive motors (Hanson and Whiteheart, 2005; Kelch, 2016). 558 mechanism, our structures clearly indicate that yeast RFC uses 618 We note that conformational changes that we observe here in 559 the much more simple direct binding model.

619 RFC appear to be more dramatic than those typically seen during 560 Once DNA is bound, PCNA must close around the ring before 620 motor function. This is likely because the constraints imposed by 561 ejection of the RFC complex (Liu et al., 2017; Marzahn et al., 2014; 621 ring closure limits the types of motions that are available. On the 562 Sakato et al., 02/2012), which we observe in the transition from 622 other hand, the open nature of the RFC complex is less 563 DNA $^{\text {PCNA-open }}$ and DNA ${ }^{\text {PCNA-closed }}$ structures. Both structures show 623 constrained and so can adopt more dramatic conformational 564 no evidence of ATP hydrolysis, suggesting that PCNA can close 624 changes. We further note that these types of large conformational 565 before ATP hydrolyzes. In contrast, it had been previously 625 changes are more commonplace in other members of the 566 hypothesized that ATP hydrolysis occurs before clamp closure 626 Initiator/Loader class of AAA+ machines. We propose that the 567 (Sakato et al., 02/2012). While DNA ${ }^{\text {PCNA-open }}$ and DNA ${ }^{\text {PCNA-closed }}$ have 627 open nature of this class provides larger conformational variability 568 similar overall interaction areas, PCNA interacts with DNA much 628 that is necessary for the regulation of these switch-like machines. 569 more intimately in the DNA ${ }^{\text {PCNA-closed }}$ structure, with direct contact

570 to several conserved basic residues lining the PCNA inner pore. 629 MATERIALS AND METHODS

571 Lys20, Arg80, and Arg147 in particular show close interaction with

572 the PCNA ring. These residues have been independently 630 Protein Purification

573 identified as critical for efficient DNA binding, ATP hydrolysis and

574 clamp loading (McNally et al., 2010; Zhou and Hingorani, 2012). 575 Therefore, PCNA is an allosteric effector in its own loading.

576 The final step in clamp loading is the hydrolysis of ATP and the 577 ejection of the clamp loader. Our structures further illuminate how 578 ATP hydrolysis is synergistically activated by PCNA and p/t-DNA. 579 While our reconstructions do not have definitive density for 580 unambiguous localization of side chains in the active sites across 581 all the structures, we observe that the interactions between 582 adjacent $A A A+$ modules become tighter upon binding both PCNA

631 RFC was purified as described previously with minor 632 modifications (Finkelstein et al., 08/2003). pET(11a)-RFC[2+3+4] 633 and pLANT-2/RIL-RFC[1+5] were transformed into BL21(DE3) $E$. 634 coli cells (Millipore). After preculture, transformants were grown in 6354 liter of prewarmed terrific broth medium supplemented with 50 $636 \mu \mathrm{g} / \mathrm{mL}$ kanamycin and $100 \mu \mathrm{g} / \mathrm{mL}$ ampicillin at $37^{\circ} \mathrm{C}$ and induced 637 with IPTG at an optical density of 0.8 . Protein expression was 638 continued at $18^{\circ} \mathrm{C}$ for 15 hours. Cells were pelleted and 
639 resuspended in $300 \mathrm{ml} \mathrm{lysis} \mathrm{buffer} \mathrm{(30} \mathrm{mM} \mathrm{Hepes-NaOH} \mathrm{pH} \mathrm{7.5,} 694$ Electron Microscopy

$640250 \mathrm{mM} \mathrm{NaCl}, 0.25 \mathrm{mM}$ EDTA, $5 \%$ glycerol, $2 \mathrm{mM} \mathrm{DTT}, 2 \mu \mathrm{g} / \mathrm{mL}$

641 aprotinin, $0.2 \mu \mathrm{g} / \mathrm{mL}$ pepstatin, $2 \mu \mathrm{g} / \mathrm{mL}$ leupeptin, $1 \mathrm{mM}$ PMSF). 695 Negative-Staining EM

642 RFC was purified by chromatography over a $10 \mathrm{~mL}$ SP-Sepharose $696100 \mathrm{nM}$ of RFC:PCNA was applied on carbon-coated 400-mesh 643 column ( $80 \mathrm{~mL}$ gradient of $300-600 \mathrm{mM} \mathrm{NaCl}$ in BufferC) and a $696100 \mathrm{nM}$ grids. Excess sample was blotted from the grid surface, the grids $64410 \mathrm{~mL}$ Q-Sepharose column $(40 \mathrm{~mL}$ gradient of $150-500 \mathrm{mM} \mathrm{NaCl} 698$ were washed twice with $50 \mathrm{mM} \mathrm{Hepes} \mathrm{pH} 7.5$ and stained with 645 in Buffer C, GEHealthcare). Peak fractions of hRFC were pooled $6991 \%$ uranyl acetate. RFC:PCNA was imaged on a $120 \mathrm{kV}$ Philips 646 and dialyzed overnight into a buffer with $30 \mathrm{mM}$ Hepes- $\mathrm{NaOH}$ pH $700 \mathrm{CM}-120$ microscope fitted with a Gatan Orius SC1000 detector. $6477.5,250 \mathrm{mM} \mathrm{NaCl}, 5 \%$ glycerol, and $2 \mathrm{mM}$ DTT.

701

648 PCNA was purified as described previously with modifications ${ }^{702}$ Cryo-EM sample preparation

649 (McNally et al., 2010). BL21(DE3) E. coli cells were transformed 703 Quantifoil R 0.6/1 (DNA dataset) grids were washed with ethyl 650 with a pET-28 vector that encodes PCNA with a PrecissionTM 704 acetate. Quantifoil and C-flat grids (Electron Microscopy 651 protease cleavable $\mathrm{N}$-terminal 6 -His tag. After transformation, 705 Sciences) were glow discharged with Pelco easiGlow for 60 s at 652 preculture and induction, 1 liter of cells was grown overnight at $1870625 \mathrm{~mA}$ (negative polarity). $2.8-3 \mu \mathrm{L}$ sample was applied to grids $653{ }^{\circ} \mathrm{C}$ in terrific broth medium supplemented with $50 \mu \mathrm{g} / \mathrm{mL} 707$ at $10^{\circ} \mathrm{C}$ and $95 \%$ humidity in a Vitrobot Mark IV (FEl). Samples 654 kanamycin. Cells were pelleted and resuspended $30 \mathrm{mM}$ Hepes 708 were blotted with a force of 5 for $5 \mathrm{~s}$ after a $2 \mathrm{~s}$ wait and plunged $655 \mathrm{pH} 7.6,20 \mathrm{mM}$ imidazole, $500 \mathrm{mM} \mathrm{NaCl}, 10 \%$ glycerol, and $5 \mathrm{mM} 709$ into liquid ethane.

656 b-mercaptoethanol. The cells were lysed, centrifuged and the 710

657 filtered lysate was applied to a $5 \mathrm{~mL}$ HisTrap FF column 711 Cryo-EM data collection

658 (GEHealthcare). The column was washed with a buffer at $1 \mathrm{M} 712$ RFC:PCNA was imaged on a Titan Krios operated at $300 \mathrm{kV}$ and $659 \mathrm{NaCl}$, and subsequently washed with a buffer at a low salt 713 equipped with an GIF energy filter at $130000 \times$ magnification and 660 concentration $(50 \mathrm{mM} \mathrm{NaCl})$. PCNA was eluted with a step of 50714 a pixel size of $0.53 \AA$ using a K2 Summit detector in super$661 \%$ with $500 \mathrm{mM}$ imidazole. The eluted protein was cleaved with 715 resolution counting mode. The data was collected in four sessions 662 Precission $^{\mathrm{TM}}$ protease for 2 hours at room temperature and 716 with a target defocus range of -1.1 to -2.4 and a total exposure of 663 applied to a $5 \mathrm{~mL}$ HiTrap Q HP column (GE Healthcare). Protein $717 \sim 49-51$ e-/ $\AA^{2}$ per micrograph averaging 50 frames. Image shift 664 was eluted from the Q HP column with a $2 \mathrm{M} \mathrm{NaCl}$ buffer in a 100718 was used to record three images per hole with SerialEM $665 \mathrm{~mL}$ gradient. Peak fractions were dialyzed against buffer 719 (Mastronarde, 2003). Defective micrographs were discarded 666 containing $30 \mathrm{mM}$ Tris pH 7.5, $100 \mathrm{mM} \mathrm{NaCl}$, and $2 \mathrm{mM} \mathrm{DTT} .720$ leaving a total of 6109 micrographs for processing. 667 Purified proteins were concentrated with an Amicon 721 RFC:PCNA:DNA was imaged on a Titan Krios operated at 300 kV 668 concentration device, aliquoted and frozen in liquid nitrogen for 722 at $81000 \times$ magnification and a pixel size of $0.53 \AA$ with a K3 669 storage at $-80^{\circ}$.

\section{Crosslinking and Mass Spectrometry}

723 detector in super-resolution mode. A total of 4499 micrographs 724 were collected in one day with a target defocus of -1.2 to -2.3 and 725 a total exposure of $\sim 40$ e-/ $/ \AA^{2}$ per micrograph averaging 30 frames.

671 RFC and PCNA were mixed in a 1/1 ratio and gel filtered into 1726

$672 \mathrm{mM}$ TCEP, $200 \mathrm{mM} \mathrm{NaCl}, 25 \mathrm{mM}$ Hepes-NaOH, pH 7.5, and 4727 Data Processing

$673 \mathrm{mM} \mathrm{MgCl}_{2}$. The protein complex was diluted to $3 \mu \mathrm{M}$ and after the 728 Micrograph frames were aligned in IMOD (Kremer et al., 1996) 674 addition of $1 \mathrm{mM}$ ATPyS and $3 \mathrm{~min}$ incubation, $1 \mathrm{mM}$ of 729 with $2 x$ binning, resulting in a pixel size of $1.06 \AA$ pixel. Initial CTF 675 Bis(sulfosuccinimidyl)suberate (BS3, Thermo Scientific Pierce) 730 estimation and particle picking was performed using cisTEM 676 was added for crosslinking. For crosslinking of DNA-bound 731 (Grant et al., 2018; Rohou and Grigorieff, 2015). Following particle 677 RFC:PCNA, 1 mM ATPyS was added to the protein complex first 732 picking, particles were extracted with a box size of 240 pixels and 678 and incubated for 2 min. $7 \mu \mathrm{M}$ primer/template DNA was added 733 subjected to 2D classification into 100 classes. Particles from 679 and incubated for another $1 \mathrm{~min}$. The primer sequence was $5^{\prime}-734$ classes with well defined features were selected for processing in 680 GCAGACACTACGAGTACATA-3' and the template sequence 735 Relion (Figures S2A,B and S3A,B). Coordinates and combined 681 was 5 '-TाTाTाTाATGTACTCGTAGTGTCTGC-3'. 736 micrographs were imported into Relion 3.0 .2 (Zivanov et al., 682 Crosslinking was started with $1 \mathrm{mM} \mathrm{BS3}$, incubated for 15 min at 737 2018), CTF parameters were re-estimated with Gctf1.06 (Zhang, 683 room temperature, and neutralized with Tris- $\mathrm{HCl}$. 7382016 ) and particles were subjected to several rounds of $3 \mathrm{D}$ 684 Sample without DNA was analyzed by mass spectrometry. The 739 classification (Figures S2D and S3C). For 3D classification of the 685 sample was reduced, alkylated, and loaded onto SDS-PAGE gel. 740 RFC:PCNA dataset, an ab initio model was generated with 686 The gel band corresponding to the crosslinked complex $>150 \mathrm{kDa} 741$ cisTEM, down-filtered to $50 \AA$ and used as reference (Figure S2C). 687 was excised, destained, and incubated with trypsin. The digested 742 For 3D classification of the RFC:PCNA:DNA dataset, class Open1 688 peptides were extracted and desalted as previously described 743 of the RFC:PCNA dataset was down-filtered to $60 \AA$ and used as 689 (Peled et al., 2018) and analyzed with LC-MS coupled to a 744 reference. Selected, well resolved 3D classes were refined with 690 ThermoFisher Scientific Q Exactive Mass Spectrometer in data 745 Relion. The cryo-EM density was postprocessed in Relion for 691 dependent mode selecting only precursors of 3 . The data was 746 estimating the resolution and density modified with PHENIX for 692 searched against the UniProt database, using Byonic and XlinkX 747 model building and refinement (Terwilliger et al., 2020 Table S2). 693 of the Proteome Discoverer 2.3 package.

\section{8}

749 Model Building and Refinement 
750 The crystal structure of yeast RFC bound to PCNA (PDB ID: 1SXJ) 804 Hingorani for providing us with the RFC expression plasmids. 751 was used for initial fitting of Autoinhibited1. All subunits were split $805 \mathrm{We}$ thank J. Andrade and Dr. B. Ueberheide for analyzing the 752 into globular domains and fitted into the cryo-EM density with 806 crosslinked sample with mass spectrometry. We thank members 753 UCSF Chimera (Pettersen et al., 2004). The model was adjusted 807 of the Kelch, Royer, and Schiffer labs for helpful discussions. 754 in Coot (Emsley and Cowtan, 2004), and real-space iteratively 808 This work was funded by NIGMS (R01-GM127776). C.G. was 755 refined with two macrocycles in PHENIX1.17 (Liebschner et al., 809 supported by an Early and Advanced Postdoc Mobility (grant 756 2019). Autoinhibited2,3 cryo-EM densities were rigid body fit with 810 numbers 168972 and 177859) Fellowship of the Swiss National 757 the refined model of Autoinhibited1, manually adjusted in coot 811 Science Foundation. G.D. was supported by LL2008 project with 758 and refined.

812 financial support from the MEYS CR as a part of the ERC CZ 759 The refined model of Autoinhibited1 from the DNA dataset (Figure 813 program.

760 S3C) was fragmented into individual subunit domains and rigid 814

761 body fitted into the cryo-EM density of Open2. The resulting 815

762 model was further flexibly fitted and refined with Namdinator 816 AUTHOR CONTRIBUTIONS

763 (Kidmose et al., 2019). The resulting model was adjusted in Coot, 817 C.G. and B.A.K. designed experiments. C.G. performed cryo-EM 764 and refined in PHENIX. The model of Open2 was used for fitting 818 sample preparations, data collection and processing with the help 765 the Open1 cryo-EM density. The fitted model was manually 819 of G.D. G.D. provided valuable insight into optimization of cryo 766 adjusted in Coot and refined in PHENIX. The cryo-EM density of 820 samples and reconstruction refinement. C.G. \& X.L., performed 767 DNA PCNA-closed (Figure S3C) was fitted using the Autoinhibited1 821 biochemical experiments. C.G., N.P.S., J.A.H. and B.A.K. built 768 model and DNA was modelled in Coot. The resulting model was 822 and C.G. refined the atomic models. C.G., X.L., J.P., and B.A.K. 769 further flexibly fitted and refined with Namdinator (Kidmose et al., 823 analyzed the data and wrote the paper with comments from 770 2019). The model was then adjusted in Coot, and refined in 824 coauthors.

771 PHENIX. The Namdinator output model of DNA ${ }^{\text {PCNA-closed }}$ was used 825

772 for fitting of the DNA ${ }^{\text {PCNA-open }}$ cryo-EM density. The fitted model 826 COMPETING INTERESTS

773 was manually adjusted in Coot and subjected to refinement in 827 The authors declare no conflict of interest.

774 PHENIX. Interface areas were analyzed with the PISA server 828

775 (Krissinel and Henrick, 2007). UCSF Chimera and Pymol were 829

776 used for figure generation (DELANO and W. L, 2002; Pettersen et 830 777 al., 2004).

\section{ATPase Assays}

$7790.3 \mu \mathrm{M}$ (Figure 6F) or $0.15 \mu \mathrm{M}$ RFC (Figure S9D) was incubated 780 with a master mix $(3 \mathrm{U} / \mathrm{mL}$ Pyruvate kinase, $3 \mathrm{U} / \mathrm{mL}$ Lactate 781 dehydrogenase, $1 \mathrm{mM}$ ATP, $670 \mu \mathrm{M}$ Phosphoenol pyruvate, 170 $782 \mu \mathrm{M}$ NADH, 50 mM Tris (pH 7.5), 0.5 mM TCEP, 5 mM MgCl, 200 $783 \mathrm{mM}$ potassium glutamate, $40 \mathrm{mM} \mathrm{NaCl}$ ), $1 \mu \mathrm{M}$ PCNA, and 784 annealed primer/template DNA ( $2 \mu \mathrm{M}$ Figure $6 \mathrm{~F}$, varying amounts 785 Figure S9D). ATPase activity was measured at $25^{\circ} \mathrm{C}$ with the 7862014 EnVison $®$ Multilabel Plate Reader to detect NAD+. Rates 787 were obtained from a linear fit of the slopes using GraphPad 788 Prism.

\section{2-AP Fluorescence}

790 2-Aminopurine (2AP) fluorescent samples were excited at $315 \mathrm{~nm}$ 791 ( $5 \mathrm{~mm}$ slit width), and emission was detected at $370 \mathrm{~nm}$ (7 mm slit 792 width) with a FluoroMax 4 (Horiba Join Yvon Inc). Reactions 793 contained 150 or $375 \mathrm{nM}$ annealed DNA and 0.5 or $1 \mu \mathrm{M}$ RFC in 794 a buffer with $50 \mathrm{mM}$ Hepes- $\mathrm{NaOH}$ pH 7.5, $200 \mathrm{mM} \mathrm{NaCl}, 4 \mathrm{mM}$ $795 \mathrm{MgCl}_{2}, 1 \mathrm{mM}$ TCEP and were carried out at room temperature. 796 Experiments Figure $6 \mathrm{C}$ were performed in the presence of $375 \mathrm{nM}$ 797 DNA, $0.5 \mu \mathrm{M}$ RFC, and $2.5 \mu \mathrm{M}$ PCNA. Experiments Figure 6D and $798 \mathrm{E}$ were performed with $150 \mathrm{nM}$ DNA, $1 \mu \mathrm{M}$ RFC, and $2.5 \mu \mathrm{M}$ 799 PCNA.

\section{ACKNOWLEDGMENTS}

801 The authors thank Drs. C. Xu, KK Song, and K. Lee for 802 assistance with data collection, and Drs. C. Xu, and A. Jecrois 803 for advice on data processing. Additionally, we thank Dr. M. 


\section{REFERENCES}

832 Bowman, G.D., O’Donnell, M., and Kuriyan, J. (2004). Structural 833 analysis of a eukaryotic sliding DNA clamp-clamp loader 834 complex. Nature 429, 724-730.

835 Cai, J., Yao, N., Gibbs, E., Finkelstein, J., Phillips, B., O’Donnell, 836 M., and Hurwitz, J. (1998). ATP hydrolysis catalyzed by human 837 replication factor $C$ requires participation of multiple subunits. 838 Proceedings of the National Academy of Sciences 95, 1160783911612.

840 Chen, S., Levin, M.K., Sakato, M., Zhou, Y., and Hingorani, M.M. 841 (5/2009). Mechanism of ATP-Driven PCNA Clamp Loading by S. 842 cerevisiae RFC. J. Mol. Biol. 388, 431-442.

843 DELANO, and W. L (2002). The PyMOL Molecular Graphics 844 System. Http://www.pymol.org.

845 Dephoure, N., Zhou, C., Villén, J., Beausoleil, S.A., Bakalarski, 846 C.E., Elledge, S.J., and Gygi, S.P. (2008). A quantitative atlas of 847 mitotic phosphorylation. Proc. Natl. Acad. Sci. U. S. A. 105, 848 10762-10767.

849 Emsley, P., and Cowtan, K. (2004). Coot: model-building tools 850 for molecular graphics. Acta Crystallogr. D Biol. Crystallogr. 60, $8512126-2132$.

852 Erzberger, J.P., and Berger, J.M. (2006). Evolutionary 853 relationships and structural mechanisms of AAA+ proteins. 854 Annu. Rev. Biophys. Biomol. Struct. 35, 93-114.

855 Finkelstein, J., Antony, E., Hingorani, M.M., and O’Donnell, M. 856 (08/2003). Overproduction and analysis of eukaryotic 857 multiprotein complexes in Escherichia coli using a dual-vector 858 strategy. Anal. Biochem. 319, 78-87.

859 Frey, M.W., Sowers, L.C., Millar, D.P., and Benkovic, S.J. (1995). 860 The nucleotide analog 2-aminopurine as a spectroscopic probe 861 of nucleotide incorporation by the Klenow fragment of 862 Escherichia coli polymerase I and bacteriophage T4 DNA 863 polymerase. Biochemistry 34, 9185-9192.

864 Gaubitz, C., Liu, X., Magrino, J., Stone, N.P., Landeck, J., 865 Hedglin, M., and Kelch, B.A. (2020b). Structure of the human 866 clamp loader reveals an autoinhibited conformation of a 867 substrate-bound AAA switch. Proceedings of the National 868 Academy of Sciences 117, 23571-23580.

869 Gerlach, P., Schuller, J.M., Bonneau, F., Basquin, J., Reichelt, 870 P., Falk, S., and Conti, E. (2018). Distinct and evolutionary 871 conserved structural features of the human nuclear exosome 872 complex. Elife 7.

873 Goedken, E.R., Levitus, M., Johnson, A., Bustamante, C., 874 O'Donnell, M., and Kuriyan, J. (2004). Fluorescence 875 measurements on the E.coli DNA polymerase clamp loader: 876 implications for conformational changes during ATP and clamp
877 binding. J. Mol. Biol. 336, 1047-1059.

878 Gomes, X.V., Schmidt, S.L., and Burgers, P.M. (2001). ATP 879 utilization by yeast replication factor C. II. Multiple stepwise ATP 880 binding events are required to load proliferating cell nuclear 881 antigen onto primed DNA. J. Biol. Chem. 276, 34776-34783.

882 Grant, T., Rohou, A., and Grigorieff, N. (2018). cisTEM, user883 friendly software for single-particle image processing. Elife 7.

884 Guenther, B., Onrust, R., Sali, A., O’Donnell, M., and Kuriyan, J. 885 (1997). Crystal structure of the delta' subunit of the clamp-loader 886 complex of E. coli DNA polymerase III. Cell 91, 335-345.

887 Hanson, P.I., and Whiteheart, S.W. (2005). AAA+ proteins: have 888 engine, will work. Nat. Rev. Mol. Cell Biol. 6, 519-529.

889 Jean, J.M., and Hall, K.B. (2001). 2-Aminopurine fluorescence 890 quenching and lifetimes: role of base stacking. Proc. Natl. Acad. 891 Sci. U. S. A. 98, 37-41.

892 Jeruzalmi, D., O’Donnell, M., and Kuriyan, J. (2001a). Crystal 893 structure of the processivity clamp loader gamma (gamma) 894 complex of E. coli DNA polymerase III. Cell 106, 429-441.

895 Jeruzalmi, D., Yurieva, O., Zhao, Y., Young, M., Stewart, J., 896 Hingorani, M., O’Donnell, M., and Kuriyan, J. (2001b). 897 Mechanism of processivity clamp opening by the delta subunit 898 wrench of the clamp loader complex of E. coli DNA polymerase 899 III. Cell 106, 417-428.

900 Jessop, M., Felix, J., and Gutsche, I. (2021). AAA+ ATPases: 901 structural insertions under the magnifying glass. Curr. Opin. 902 Struct. Biol. 66, 119-128.

903 Johnson, A., Yao, N.Y., Bowman, G.D., Kuriyan, J., and 904 O’Donnell, M. (2006). The Replication Factor C Clamp Loader 905 Requires Arginine Finger Sensors to Drive DNA Binding and 906 Proliferating Cell Nuclear Antigen Loading. J. Biol. Chem. 281, 907 35531-35543.

908 Kelch, B.A. (2016). Review: The lord of the rings: Structure and 909 mechanism of the sliding clamp loader. Biopolymers 105, 532910546.

911 Kelch, B.A., Makino, D.L., O’Donnell, M., and Kuriyan, J. (2011). 912 How a DNA polymerase clamp loader opens a sliding clamp. 913 Science 334, 1675-1680.

914 Kelch, B.A., Makino, D.L., O’Donnell, M., and Kuriyan, J. (2012). 915 Clamp loader ATPases and the evolution of DNA replication 916 machinery. BMC Biol. 10, 34-48.

917 Kidmose, R.T., Juhl, J., Nissen, P., Boesen, T., Karlsen, J.L., and 918 Pedersen, B.P. (2019). Namdinator - automatic molecular 919 dynamics flexible fitting of structural models into cryo-EM and 920 crystallography experimental maps. IUCrJ 6, 526-531. 
921 Kim, H.S., and Brill, S.J. (2001). Rfc4 interacts with Rpa1 and is 922 required for both DNA replication and DNA damage checkpoints 923 in Saccharomyces cerevisiae. Mol. Cell. Biol. 21, 3725-3737.

924 Kremer, J.R., Mastronarde, D.N., and McIntosh, J.R. (1996). 925 Computer visualization of three-dimensional image data using 926 IMOD. J. Struct. Biol. 116, 71-76.

927 Krissinel, E., and Henrick, K. (2007). Inference of 928 Macromolecular Assemblies from Crystalline State. Journal of 929 Molecular Biology 372, 774-797.

930 Liebschner, D., Afonine, P.V., Baker, M.L., Bunkóczi, G., Chen, 931 V.B., Croll, T.I., Hintze, B., Hung, L.W., Jain, S., McCoy, A.J., et 932 al. (2019). Macromolecular structure determination using X-rays, 933 neutrons and electrons: recent developments in Phenix. Acta 934 Crystallogr D Struct Biol 75, 861-877.

935 Liu, J., Zhou, Y., and Hingorani, M.M. (2017). Linchpin DNA936 binding residues serve as go/no-go controls in the replication 937 factor C-catalyzed clamp loading mechanism. J. Biol. Chem. 938 jbc.M117.798702.

939 Majka, J., and Burgers, P.M.J. (2004). The PCNA-RFC families of 940 DNA clamps and clamp loaders. Prog. Nucleic Acid Res. Mol. 941 Biol. 78, 227-260.

942 Marzahn, M.R., Hayner, J.N., Meyer, J.A., and Bloom, L.B. 943 (2014). Kinetic analysis of PCNA clamp binding and release in 944 the clamp loading reaction catalyzed by Saccharomyces 945 cerevisiae replication factor C. Biochim. Biophys. Acta 1854, $31-$ 94638.

947 Mastronarde, D.N. (2003). SerialEM: A Program for Automated 948 Tilt Series Acquisition on Tecnai Microscopes Using Prediction 949 of Specimen Position. Microsc. Microanal. 9, 1182-1183.

950 McNally, R., Bowman, G.D., Goedken, E.R., O'Donnell, M., and 951 Kuriyan, J. (2010). Analysis of the role of PCNA-DNA contacts 952 during clamp loading. BMC Struct. Biol. 10, 3.

953 Moldovan, G.-L., Pfander, B., and Jentsch, S. (2007). PCNA, the 954 maestro of the replication fork. Cell 129, 665-679.

955 Nakane, T., Kimanius, D., Lindahl, E., and Scheres, S.H.W. 956 (2018). Characterisation of molecular motions in cryo-EM single957 particle data by multi-body refinement in RELION. Elife 7 .

958 Ochoa, D., Jarnuczak, A.F., Viéitez, C., Gehre, M., Soucheray, 959 M., Mateus, A., Kleefeldt, A.A., Hill, A., Garcia-Alonso, L., Stein, 960 F., et al. (2020). The functional landscape of the human 961 phosphoproteome. Nat. Biotechnol. 38, 365-373.

962 O'Donnell, M., Jeruzalmi, D., and Kuriyan, J. Clamp loader 963 structure predicts the architecture of DNA polymerase III 964 holoenzyme and RFC. Curr. Biol. 11, R935-R946.

965 Olsen, J.V., Vermeulen, M., Santamaria, A., Kumar, C., Miller,
966 M.L., Jensen, L.J., Gnad, F., Cox, J., Jensen, T.S., Nigg, E.A., et 967 al. (2010). Quantitative phosphoproteomics reveals widespread 968 full phosphorylation site occupancy during mitosis. Sci. Signal. 969 3, ra3.

970 Peled, M., Tocheva, A.S., Sandigursky, S., Nayak, S., Philips, 971 E.A., Nichols, K.E., Strazza, M., Azoulay-Alfaguter, I., Askenazi, 972 M., Neel, B.G., et al. (2018). Affinity purification mass 973 spectrometry analysis of PD-1 uncovers SAP as a new 974 checkpoint inhibitor. Proc. Natl. Acad. Sci. U. S. A. 115, E468975 E477.

976 Pettersen, E.F., Goddard, T.D., Huang, C.C., Couch, G.S., 977 Greenblatt, D.M., Meng, E.C., and Ferrin, T.E. (2004). UCSF 978 Chimera--a visualization system for exploratory research and 979 analysis. J. Comput. Chem. 25, 1605-1612.

980 Rohou, A., and Grigorieff, N. (2015). CTFFIND4: Fast and 981 accurate defocus estimation from electron micrographs. J. 982 Struct. Biol. 192, 216-221.

983 Sakato, M., Zhou, Y., and Hingorani, M.M. (02/2012). ATP 984 Binding and Hydrolysis-Driven Rate-Determining Events in the 985 RFC-Catalyzed PCNA Clamp Loading Reaction. J. Mol. Biol. 986 416, 176-191.

987 Sakato, M., O'Donnell, M., and Hingorani, M.M. (2012). A central 988 swivel point in the RFC clamp loader controls PCNA opening 989 and loading on DNA. J. Mol. Biol. 416, 163-175.

990 Shiomi, Y., Usukura, J., Masamura, Y., Takeyasu, K., Nakayama, 991 Y., Obuse, C., Yoshikawa, H., and Tsurimoto, T. (2000). ATP992 dependent structural change of the eukaryotic clamp-loader 993 protein, replication factor C. Proc. Natl. Acad. Sci. U. S. A. 97, 994 14127-14132.

995 Simonetta, K.R., Kazmirski, S.L., Goedken, E.R., Cantor, A.J., 996 Kelch, B.A., McNally, R., Seyedin, S.N., Makino, D.L., O'Donnell, 997 M., and Kuriyan, J. (05/2009). The Mechanism of ATP-

998 Dependent Primer-Template Recognition by a Clamp Loader 999 Complex. Cell 137, 659-671.

1000 Terwilliger, T.C., Ludtke, S.J., Read, R.J., Adams, P.D., and 1001 Afonine, P.V. (2020). Improvement of cryo-EM maps by density 1002 modification. Nat. Methods 17, 923-927.

1003 Thompson, J.A., Marzahn, M.R., O'Donnell, M., and Bloom, L.B. 1004 (2012). Replication Factor C Is a More Effective Proliferating Cell 1005 Nuclear Antigen (PCNA) Opener than the Checkpoint Clamp 1006 Loader, Rad24-RFC. J. Biol. Chem. 287, 2203-2209.

1007 Tomida, J., Masuda, Y., Hiroaki, H., Ishikawa, T., Song, I., 1008 Tsurimoto, T., Tateishi, S., Shiomi, T., Kamei, Y., Kim, J., et al. 1009 (2008). DNA damage-induced ubiquitylation of RFC2 subunit of 1010 replication factor C complex. J. Biol. Chem. 283, 9071-9079.

1011 Wang, X., Li, J., Schowalter, R.M., Jiao, J., Buck, C.B., and You, $1012 \mathrm{~J}$. (2012). Bromodomain protein Brd4 plays a key role in Merkel 1013 cell polyomavirus DNA replication. PLoS Pathog. 8, e1003021. 
1014 Wang, X., Helfer, C.M., Pancholi, N., Bradner, J.E., and You, J. 1024 Zhou, Y., and Hingorani, M.M. (2012). Impact of Individual 1015 (2013). Recruitment of Brd4 to the human papillomavirus type 161025 Proliferating Cell Nuclear Antigen-DNA Contacts on Clamp 1016 DNA replication complex is essential for replication of viral DNA. 1026 Loading and Function on DNA. J. Biol. Chem. 287, 353701017 J. Virol. 87, 3871-3884. 102735381.

1018 Watson, J.D. (2014). Molecular Biology of the Gene (Pearson). 1028 Zhuang, Z., Yoder, B.L., Burgers, P.M.J., and Benkovic, S.J. 1029 (2006). The structure of a ring-opened proliferating cell nuclear 1019 Yoo, J., Wu, M., Yin, Y., Herzik, M.A., Lander, G.C., and Lee, S.- 1030 antigen-replication factor C complex revealed by fluorescence 1020 Y. (2018). Cryo-EM structure of a mitochondrial calcium 1031 energy transfer. Proc. Natl. Acad. Sci. U. S. A. 103, 2546-2551. 1021 uniporter. Science eaar4056.

1022 Zhang, K. (2016). Gctf: Real-time CTF determination and 1032 Zivanov, J., Nakane, T., Forsberg, B.O., Kimanius, D., Hagen, 1033 W.J., Lindahl, E., and Scheres, S.H.W. (2018). New tools for 1023 correction. J. Struct. Biol. 193, 1-12. 1034 automated high-resolution cryo-EM structure determination in 1035 RELION-3. Elife 7. 